\title{
Laying the foundation for a taxonomic review of Puccinia coronata s.l. in a phylogenetic context
}

\author{
Miao Liu • Sarah Hambleton
}

Received: 4 November 2011 /Revised: 24 February 2012 / Accepted: 7 March 2012 / Published online: 18 April 2012

(C) The Author(s) 2012. This article is published with open access at Springerlink.com

\begin{abstract}
Intra-specific classification of Puccinia coronata has been controversial, with previous approaches falling into three major categories: 1 . A two-species system, namely $P$. coronifera and P. coronata; 2 . The same two-species system subdivided into many formae speciales, in which the host range of each is restricted to species within one genus of Poaceae; 3. A one-species system, P. coronata, subdivided into a few varieties with host ranges that may overlap. To re-assess these concepts in the context of multigene analyses and comparative morphological assessments, data were generated for a comprehensive set of herbarium and recently collected specimens, representing a broad range of hosts and geographic origins. Phylogenetic analyses of a combined data set of DNA sequences for four loci (BT, COI, ITS, and RPB2) revealed a high degree of genetic variation. Morphological differences among phylogenetic lineages were overlapping but nine lineages were differentiated using calculated means for teliospore and urediniospore length/width as well as measurements for the teliospore hilum and digitation. The taxon infecting Avena also comprises collections from a wide range of other grass hosts while other lineages, such as those on Bromus and
\end{abstract}

M. Liu $\cdot$ S. Hambleton $(\bowtie)$

Biodiversity (Mycology and Botany), Eastern Cereal and Oilseed

Research Centre, Agriculture and Agri-Food Canada,

960 Carling Ave.,

Ottawa K1A 0C6, Canada

e-mail: sarah.hambleton@agr.gc.ca

M. Liu

e-mail: miao.liu@ars.usda.gov

Present Address:

M. Liu

Cereal Disease Laboratories,

1551 Lindig St.

Saint Paul, MN 55108, USA
Agrostis, were restricted in host association. Type specimen DNA sequences included in the analyses resolved the placement of five previously described varieties. Based on evidence of host specificity, morphology and multigene analyses, we recognized seven species, one of which was further divided into two varieties. Expanded descriptions, illustrations and a synoptic key are provided. A new series, Puccinia Series Coronata, was erected to accommodate all the lineages comprising P. coronata sensu lato.

Keywords Crown rust · Uredinales · Phylogeny · Cryptic species $\cdot$ Variety $\cdot$ Forma specialis

\section{Introduction}

The telial stage of crown rust, Puccinia coronata, is readily identified based on the distinctive, yet morphologically variable, digitations adorning the upper cell of the teliospore hence, the common name. Although well-known as causing disease on oats, the species also occurs on a broad range of other hosts, and is considered one of the most important and damaging diseases worldwide of cultivated oat (Avena sativa) and pasture grasses (Harder and Haber 1992; Potter et al. 1990; Simons 1970). In Canada, control of the disease mainly depends on the deployment of resistant cultivars, assisted by early planting strategies and fungicide applications (McCallum et al. 2007). For many years breeding for resistance was successful in providing a series of new cultivars with good rust resistance, but nearly all of the effective resistance genes used to date have now been overcome (for review see McCallum et al. 2007). In contrast to the detrimental effects to agriculture, $P$. coronata has also been investigated for beneficial purposes, such as a potential 
biocontrol agent of wild oats (Av. fatua) on San Clemente Island, California (Carsten et al. 2000).

The macrocyclic lifecycle of the species includes uredinial and telial stages on a diverse range of grass species occurring worldwide and spanning more than 45 genera of Poaceae (Cummins 1971). Pycnia and aecia occur on shrubs in the buckthorn family (Rhamnaceae), which are widely distributed in the Northern Hemisphere and Middle East (Roelfs 1985). The future implementation of targeted disease management and breeding efforts, and the robust assessment of biological control potential, depend on a deeper understanding of the genetic and biological variation within this species complex.

Puccinia coronata was first described by Corda in 1837 from Reichenberg (now Liberec, Czech Republic) on Luzula albida (later redetermined as Calamagrostis arundinaceae or Cal. villosa). Early infection experiments using teliospores from a range of grasses to inoculate two aecial shrub hosts, Rhamnus frangula (the current name Frangula alnus is used in the following paragraphs; GRIN, http://www.ars-grin.gov/ cgi-bin/npgs/html/tax_search.pl, Aug 2010) and R. cathartica, resulted in infection of one or the other host, but never both (Cornu 1880; Eriksson 1894; Klebahn 1892; Muhlethaler 1911; Nielsen 1877). Based on the initial evidence, Klebahn (1894) divided P. coronata into two species, i.e. P. coronata on Fr. alnus and P. coronifera on $R$. cathartica. Crown rust of oats (Av. sativa) was classified in P. coronifera because isolates did not infect $F r$. alnus (Eriksson 1894; Muhlethaler 1911). In the same year, having recognized several specialized forms of crown rust, Eriksson (1894) divided them into four series, each series including multiple formae speciales (f. spp.) whereby each forma specialis (f. sp.) was restricted to a specific telial host genus. Later, Mühlethaler (1911) proposed different groupings based on his infection experiments. Nevertheless, the two species of Klebahn (1894) were accepted as distinct groups in both the Eriksson (1894) and Mühlethaler (1911) systems. These and subsequent studies resulted in the recognition of $13 \mathrm{f}$. spp.: agropyri, agrostis, avenae, alopecuri, bromi, calamagrostis, festucae, glyceriae, holci, lolii, melicae, phalaridis and secalis (Brown 1937; Eriksson 1894, 1908; Melhus et al. 1922; Peturson 1954).

The appropriateness of the two species system was questioned by Melhus et al. (1922) and Dietz (1926) because of studies demonstrating cross-infection between $P$. coronata and $P$. coronifera and their respective hosts. Melhus et al. (1922) reviewed experimental data published by Treboux $(1912,1914)$ indicating that aecial spores from $F r$. alnus infected Av. sativa, while those from R. cathartica infected Agrostis stolonifera, Cal. arundinacea, and Phalaris arundinacea, documented hosts of $P$. coronata not $P$. coronifera. Eshed and Dinoor (1980) noted that during this period of time, the concept of forma specialis broadened from implying a pathogenic form specific for one host genus to include specificity for multiple genera, which may overlap.

In the early 1930s, Fraser and Ledingham (1933) completed a comprehensive study of crown rust in Canada, observing that the aecial state occurred on only four species of shrubs in the Prairie Provinces, i.e. R. cathartica, $R$. alnifolia, Lepargyraea canadensis and Elaeagnus commutata. Based on aecial (I) characteristics (absence or presence of hypertrophy of the tissue, arrangement of aecial cups and color of aecia), and in vitro inoculation experiments with various grasses to document development of the uredinial (II) and telial (III) stages, four varieties were designated: "Puccinia coronata Avenae" (aecial state I on R. cathartica, II and III on Avena spp.), "Puccinia coronata Calamagrostis" (I on R. alnifolia, II and III mainly on Calamagrostis spp. and Scolochloa festucacea), "Puccinia coronata Bromi" (I on L. canadensis [current name: Shepherdia canadensis, GRIN Aug 2010], II and III on Bromus spp.), "Puccinia coronata Elaeagni" (I on E. commutata, II and III on Cal. elongata [name was not found in GRIN]).

In the early 1970s, Cummins (1971) published a new classification based on detailed studies that focused on the minute morphological differences among urediniospores and teliospores and de-emphasized host association. Five varieties were recognized including $P$. coronata var. avenae sensu Fraser and Ledingham (1933). The other three varieties of Fraser and Ledingham (1933) were combined within the type variety, $P$. coronata var. coronata, a broad and inclusive taxon found on Berchemia, Rhamnus and Elaeagnus (I), and grasses in 10 different tribes (II, III). The three additional varieties were: $P$. coronata var. gibberosa (Lagerh.) Joerst. (on Festuca altissima in Europe); P. coronata var. rangiferina (Ito) Cumm. (on Cal. arundinacea and Cal. epigeios in China and Japan); and P. coronata var. himalensis Barcl. (on grasses in four different tribes) characterized by having small thin-walled urediniospores.

More recent research emphasizing spore morphology, geographic distribution and natural occurrence on various hosts in Europe was summarized by Urban and Marková (1993). Their classification divided the species complex into three varieties: $P$. coronata var. coronata (I on $R$. cathartica and $F r$. alnus, II and III on a wide range of grasses), $P$. coronata var. avenae (I commonly on Rhamnus spp., II and III mainly on Avena) and P. coronata var. intermedia (I on $R$. pumilus Turra [written as $R$. pumila Turra in the article], II and III on Calamagrostis and Sesleria). They pointed out that $P$. coronata var. avenae also likely occurs on wild oats in nature.

The contradictions among these classifications resulted from an emphasis on plastic morphological characters, insufficient sampling, uncertainties about host ranges and difficulties in confirming host alterations in the life cycle. DNA-based studies could be used to resolve some of these 
contradictions. Recent analyses by Szabo (2006) of rDNA sequences for 16 specimens provided molecular evidence that $P$. coronata is a species complex comprising multiple phylogenetic lineages. Based on those results, six subspecies were proposed but not named. Sampling was limited to seven of the 45 reported telial host genera and only one specimen on Avena was included.

The objectives of the present study were to analyze data from four gene regions for a broader sampling of $P$. coronata, including multiple representatives from a wider host and geographic range, re-assess the published classification systems, and to investigate the following questions. Is the pathogen that causes crown rust on oats a phylogenetically distinct group within the $P$. coronata complex and what is its host range? Will increased sampling reveal additional intra-specific taxa and can the lineages be differentiated morphologically? We present a framework for a new classification for the species based on a review of previous taxonomic treatments and the synthesis of molecular, morphological and biological data.

\section{Material and methods}

\section{Fungal specimens}

Fungal specimens were obtained either from herbaria or as new collections from the field. Twelve international herbaria loaned material (see Table 1 footnotes for list) and all new collections were deposited in the National Mycological Herbarium in Ottawa, Canada (DAOM). Herbarium voucher numbers, plant host, provenance, year collected and Genbank accession numbers for 156 specimens sequenced in this study are listed in Table 1.

\section{Genomic DNA extraction}

Small pieces of infected plant material, $1-4 \times 0.1-0.8 \mathrm{~cm}$, were selected for DNA extraction and surface-sterilized by gentle wiping using a Kimwipe (KimTech Inc., Louisville, USA) sprayed with $75 \%$ ethanol. For small batches of samples, E.Z.N.A. Fungal DNA Extraction Kit (VWR) was used as described in Liu and Hambleton (2010); larger batches of samples were processed in a 96-well plate format using the QIAGEN DNeasy 96 Plant Kit (QIAGEN Inc., Toronto, Canada) as per the manufacturer's protocol.

PCR amplification and sequencing

We targeted short amplicons due to the challenge to PCRamplify long fragments for DNA from dried herbarium specimens, in which the DNA degradation from poor initial drying methods as well as specimen aging could be inhibiting factors. Four gene regions were sampled. The nuclear
rDNA internal transcribed spacer (ITS) region and a portion of the $\beta$-tubulin (BT) gene, between introns 5 and 7 (Ayliffe et al. 2001), were amplified and sequenced using the method described in Liu and Hambleton (2010). Exons one and two of the mitochondrial cytochrome oxidase subunit I (COI) gene were amplified by primers P360f (GCTAAGGATA TTGCCATTCTATAT) and P360r (TCCATCCYGTYCCT GCYCC) designed by M. Allaire (http://www.boldsystems. org/views/primerlist.php), with annealing temperature at $51^{\circ} \mathrm{C}$ for $30 \mathrm{sec}$.

For the nuclear RNA Polymerase II subunit 2 (RPB2) gene, semi-nested PCR was performed using newly designed primers to amplify a fragment beginning in exon 7 downstream from primer RPB2-7 F (Liu et al. 1999). First round amplification was with primers RPB2-187f (CGATCCTGTGYTAY TCGGGMTAYAACCA) and RPB2-853r2 (GCATCRC CYTCRTTVCCKGWG), and the second round with the forward primer RPB2-492f (CGGATGAAGACRCAYACK AARCG) and the same reverse primer. Protocols were as same as those for amplifying BT (Liu and Hambleton 2010).

If no DNA fragment was amplified, the PreCR Repair Mix (New England BioLabs, Ipswich, USA) was used as per the manufacturer's instructions to attempt DNA repair, in case DNA degradation was the reason for the failed PCR amplifications.

\section{Phylogenetic analyses}

Sequences were edited using Sequencher ${ }^{\mathrm{TM}} 4.7$ (Gene Codes Corporation, Ann Arbor, USA) and compiled using BioEdit Sequence Alignment Editor 7.0.5.3 (Hall 1999). The compiled sequences were submitted to a web server (http://mafft.cbrc.jp/alignment/server/index.html) for alignment by MAFFT ver.5 (Katoh et al. 2005) and the model FFT-NS-I (iterative refinement method) was selected. The sequence alignments were manually adjusted to correct obvious misalignments by the computer algorithm.

Data matrices were subjected to parsimony analysis in PAUP* 4.0b10 (Swofford 1998). Heuristic searches with random stepwise addition of 100 replicates and tree bisection-reconnnection (TBR) branch swapping were conducted. A limit of 1,000,000 rearrangements was set for each replicate. Bootstrapping analyses were set with 1000 replicates with full heuristic search of random stepwiseaddition of 10 replicates and a limit of 10,000 rearrangements per replicate. Gaps were treated as missing data. Parsimony analyses were conducted for each locus separately and for the combined data set. In order to evaluate the level of congruence of individual loci, we estimated Partitioned Bremer Support for the branches of the tree based on the combined data set (Baker et al. 1998) using TreeRot ver. 3 (Sorenson and Franzosa 2007). 
Table 1 List of fungal specimens used in the phylogenetic analyses

\begin{tabular}{|c|c|c|c|c|c|c|c|c|c|}
\hline $\begin{array}{l}\text { Species Name } \\
\text { and Voucher } \\
\text { Number }^{\mathrm{a}}\end{array}$ & Clade & $\mathrm{PCA}^{\mathrm{b}}$ & Host & Country & Year & ITS $^{c}$ & BT & $\mathrm{COI}$ & RPB2 \\
\hline \multicolumn{10}{|c|}{ P. coronata var. avenae f. sp. avenae (Clade V): Wide host range; Cosmopolitan } \\
\hline BR 8665 & $\mathrm{~V}$ & no & $\begin{array}{l}\text { Agropyron } \\
\text { coninum }\end{array}$ & Belgium & 1993 & HM131278 & - & - & - \\
\hline DAOM 240187 & $\mathrm{~V}$ & yes & $\begin{array}{l}\text { Avena cf } \\
\text { fatua }\end{array}$ & Canada & 2006 & HM131285 & HM147304 & HM147429 & HM147374 \\
\hline K(M): 77013 & $\mathrm{~V}$ & yes & $\begin{array}{c}\text { Avena cf } \\
\text { sterilis }\end{array}$ & Greece & 2000 & HM131289 & HM147297 & HM147422 & - \\
\hline DAOM 240064 & $\mathrm{~V}$ & yes & Avena satica & Canada & 2006 & HM057140 & HM068002 & HM147405 & HM147354 \\
\hline $\begin{array}{l}\text { (S)reg.nr. } \\
\text { F34373 }\end{array}$ & $\mathrm{V}$ & no & Avena sativa & Argentina & 2000 & HM131257 & - & - & - \\
\hline $\begin{array}{l}\text { (S)reg.nr. } \\
\text { F35766 }\end{array}$ & $\mathrm{V}$ & yes & Avena sativa & Sweden & 2003 & HM131259 & HM147294 & HM147419 & HM147365 \\
\hline BP 88887 & V & no & Avena sativa & Hungary & 1988 & HM131266 & - & HM147416 & - \\
\hline BP 91484 & $\mathrm{~V}$ & yes & Avena sativa & Hungary & 1986 & HM131267 & HM147292 & HM147415 & HM147363 \\
\hline BPI 0195485 & $\mathrm{~V}$ & no & Avena sativa & Australia & 1981 & HM131269 & - & HM147418 & - \\
\hline BPI 843832 & V & no & Avena sativa & Germany & 1948 & HM131274 & - & - & - \\
\hline BPI 871766 & V & yes & Avena sativa & USA & 2004 & HM131276 & HM147293 & HM147417 & HM147364 \\
\hline $\begin{array}{l}\text { PUR } 22125 \\
\text { (LT) }\end{array}$ & V & no & Avena sativa & Canada & 1923 & HM131256 & - & - & - \\
\hline PUR N1190 & $\mathrm{V}$ & no & Avena sativa & USA & 2001 & HM131292 & HM147323 & - & - \\
\hline PUR N5418 & $\mathrm{V}$ & no & Avena sativa & Panama & 1973 & HM131300 & - & HM147390 & - \\
\hline DAOM 240180 & $\mathrm{~V}$ & no & Avena sp. & Canada & 2005 & HM131280 & HM147316 & - & - \\
\hline DAOM 240181 & $\mathrm{~V}$ & no & Avena $s p$. & Canada & 2005 & HM131281 & - & - & - \\
\hline DAOM 240182 & $\mathrm{~V}$ & no & Avena $s p$ & Canada & 2005 & HM131282 & - & - & - \\
\hline DAOM 240184 & $\mathrm{~V}$ & no & Avena $s p$ & Canada & 2006 & HM131283 & - & HM147421 & HM147370 \\
\hline DAOM 240186 & $\mathrm{~V}$ & no & Avena $s p$. & Canada & 2006 & HM131284 & HM147303 & - & - \\
\hline DAOM 240190 & $\mathrm{~V}$ & no & Avena sp. & Canada & 2006 & HM131286 & - & - & - \\
\hline PUR N1365 & $\mathrm{V}$ & no & $\begin{array}{l}\text { Dactylis } \\
\text { glomerata }\end{array}$ & USA & 1997 & HM131293 & - & HM147386 & - \\
\hline BR 47770-46 & $\mathrm{V}$ & no & $\begin{array}{l}\text { Festuca } \\
\quad \text { arundinacea }\end{array}$ & Belgium & 1994 & HM131277 & - & - & - \\
\hline PUR N1542 & V & no & Festuca sp. & USA & 1997 & HM131296 & - & - & - \\
\hline B 700012413 & $\mathrm{~V}$ & no & $\begin{array}{l}\text { Glyceria } \\
\text { maxima }\end{array}$ & Austria & 1981 & HM131260 & - & HM147428 & - \\
\hline BP 87217 & $\mathrm{~V}$ & no & $\begin{array}{l}\text { Glyceria } \\
\text { maxima }\end{array}$ & Hungary & 1980 & HM131264 & - & - & HM147358 \\
\hline BP 88886 & $\mathrm{~V}$ & no & $\begin{array}{l}\text { Glyceria } \\
\text { maxima }\end{array}$ & Hungary & 1993 & HM131265 & - & HM147410 & - \\
\hline BP 91486 & V & no & $\begin{array}{l}\text { Glyceria } \\
\text { maxima }\end{array}$ & Hungary & 1986 & HM131268 & - & - & - \\
\hline B 700012414 & V & no & Glyceria sp. & Austria & 1983 & HM131261 & - & - & - \\
\hline BPI 60255 & V & no & Hordeum sp. & Columbia & 1960 & HM131270 & - & HM147433 & - \\
\hline BP 87214 & V & no & $\begin{array}{l}\text { Lolium } \\
\quad \text { multiflorum }\end{array}$ & Hungary & 1982 & HM131263 & - & HM147411 & - \\
\hline BPI 60333 & $\mathrm{~V}$ & yes & $\begin{array}{l}\text { Lolium } \\
\text { perenne }\end{array}$ & Afghanistan & 1976 & HM131271 & HM147307 & HM147434 & - \\
\hline DAOM 181681 & V & no & $\begin{array}{l}\text { Lolium } \\
\text { perenne }\end{array}$ & Canada & 1981 & HM131279 & HM147317 & - & HM147344 \\
\hline PUR F19478 & $\mathrm{V}$ & no & $\begin{array}{l}\text { Lolium } \\
\text { perenne }\end{array}$ & Chile & 1970 & HM131290 & - & - & - \\
\hline BPI 60405 & $\mathrm{~V}$ & no & $\begin{array}{l}\text { Phalaris } \\
\text { stenoptera }\end{array}$ & Argentina & 1958 & HM131272 & - & - & - \\
\hline PUR N436 & V & no & Poa pratensis & USA & 1992 & HM131299 & - & - & - \\
\hline PUR N1982 & $\mathrm{V}$ & no & Poa sp. & USA & 1991 & HM131298 & - & - & - \\
\hline
\end{tabular}


Table 1 (continued)

\begin{tabular}{|c|c|c|c|c|c|c|c|c|c|}
\hline $\begin{array}{l}\text { Species Name } \\
\text { and Voucher } \\
\text { Number }^{\mathrm{a}}\end{array}$ & Clade & $\mathrm{PCA}^{\mathrm{b}}$ & Host & Country & Year & $\operatorname{ITS}^{\mathrm{c}}$ & BT & $\mathrm{COI}$ & RPB2 \\
\hline $\begin{array}{l}\text { (S)reg.nr. } \\
\text { F35463 }\end{array}$ & $\mathrm{V}$ & no & $\begin{array}{l}\text { Rhamnus } \\
\text { cathartica }\end{array}$ & Sweden & 2003 & HM131258 & HM147295 & - & HM147366 \\
\hline BPI 747668 & V & no & $\begin{array}{l}\text { Rhamnus } \\
\text { cathartica }\end{array}$ & USA & 1995 & HM131273 & - & - & - \\
\hline PUR N1389 & V & no & $\begin{array}{l}\text { Rhamnus } \\
\text { cathartica }\end{array}$ & USA & 1995 & HM131294 & - & - & - \\
\hline $\mathrm{K}(\mathrm{M}): 127750$ & V & no & $\begin{array}{l}\text { Schedonorus } \\
\text { gigantea }\end{array}$ & England & 1988 & HM131288 & - & HM147424 & - \\
\hline BPI 871669 & $\mathrm{~V}$ & yes & $\begin{array}{l}\text { Schedonorus } \\
\text { phoenix }\end{array}$ & USA & 2004 & HM131275 & HM147305 & HM147431 & - \\
\hline DAOM 240723 & V & no & $\begin{array}{l}\text { Schedonorus } \\
\text { phoenix }\end{array}$ & Canada & 2006 & HM131287 & HM147290 & - & - \\
\hline PUR N1008 & $\mathrm{V}$ & no & $\begin{array}{l}\text { Schedonorus } \\
\text { phoenix }\end{array}$ & USA & 1995 & HM131291 & HM147333 & - & - \\
\hline PUR N1405 & V & no & $\begin{array}{l}\text { Schedonorus } \\
\text { phoenix }\end{array}$ & USA & 1995 & HM131295 & HM147325 & HM147389 & - \\
\hline PUR N1015 & V & yes & $\begin{array}{l}\text { Schedonorus } \\
\text { phoenix }\end{array}$ & USA & 1995 & HM131301 & HM147341 & HM147402 & HM147348 \\
\hline BP 86576 & V & no & $\begin{array}{l}\text { Schedonorus } \\
\text { pratensis }\end{array}$ & Hungary & 1985 & HM131262 & - & - & - \\
\hline PUR N1977 & V & no & $\begin{array}{l}\text { Schedonorus } \\
\text { pratensis }\end{array}$ & USA & 1998 & HM131297 & HM147324 & HM147388 & - \\
\hline
\end{tabular}

P. coronata var. avenae f. sp. graminicola (Clade IV): on Arrhenatherum, Glyceria and Holcus; North America, South America, Europe

\begin{tabular}{|c|c|c|c|c|c|c|c|c|c|}
\hline BP 91761 & IV & no & $\begin{array}{l}\text { Arrhenatherum } \\
\text { elatius }\end{array}$ & Hungary & 1982 & HM131240 & - & HM147409 & - \\
\hline PUR F16064 & IV & no & $\begin{array}{l}\text { Arrhenatherum } \\
\text { elatius }\end{array}$ & $\begin{array}{l}\text { Great } \\
\text { Britain }\end{array}$ & 1958 & - & - & HM147394 & HM147380 \\
\hline PUR N5407 & IV & no & $\begin{array}{l}\text { Arrhenatherum } \\
\text { elatius }\end{array}$ & USA & 1991 & HM131253 & HM147329 & - & - \\
\hline PUR N5409 & IV & no & $\begin{array}{l}\text { Arrhenatherum } \\
\text { elatius }\end{array}$ & USA & 1990 & HM131254 & HM147330 & - & - \\
\hline K(M): 104797 & IV & yes & $\begin{array}{l}\text { Glyceria } \\
\text { maxima }\end{array}$ & England & 2002 & HM131244 & HM147298 & HM147423 & - \\
\hline B 700007213 & IV & no & Holcus lanatus & Austria & 1994 & HM131239 & - & - & - \\
\hline BPI 871068 & IV & yes & $\begin{array}{l}\text { Holcus } \\
\quad \text { lanatus }\end{array}$ & USA & 2005 & HM131243 & HM147306 & HM147432 & HM147376 \\
\hline $\mathrm{K}(\mathrm{M}): 70209$ & IV & no & $\begin{array}{l}\text { Holcus } \\
\quad \text { lanatus }\end{array}$ & England & 2000 & HM131248 & - & - & - \\
\hline PUR F19479 & IV & yes & $\begin{array}{l}\text { Holcus } \\
\text { lanatus }\end{array}$ & Argentina & 1970 & HM131249 & HM147332 & HM147392 & - \\
\hline PUR N1251 & IV & yes & $\begin{array}{l}\text { Holcus } \\
\text { lanatus }\end{array}$ & USA & 1992 & HM131250 & HM147342 & HM147403 & HM147351 \\
\hline PUR N1252 & IV & no & $\begin{array}{l}\text { Holcus } \\
\text { lanatus }\end{array}$ & USA & 1992 & HM131251 & - & - & - \\
\hline PUR N5376 & IV & no & $\begin{array}{l}\text { Holcus } \\
\quad \text { lanatus }\end{array}$ & USA & 1992 & HM131252 & - & - & - \\
\hline PUR N95 & IV & no & $\begin{array}{l}\text { Holcus } \\
\text { lanatus }\end{array}$ & Spain & 1978 & HM131255 & - & - & - \\
\hline $\mathrm{K}(\mathrm{M}): 115658$ & IV & yes & Holcus sp. & England & 2003 & HM131245 & HM147301 & HM147426 & HM147373 \\
\hline BPI 58842 & IV & no & $\begin{array}{l}\text { Rhamnus } \\
\text { cathartica }\end{array}$ & Germany & 1962 & HM131241 & - & - & - \\
\hline $\mathrm{K}(\mathrm{M}): 139492$ & IV & no & $\begin{array}{l}\text { Rhamnus } \\
\text { cathartica }\end{array}$ & England & 2006 & HM131246 & - & - & - \\
\hline $\mathrm{K}(\mathrm{M}): 139520$ & IV & no & $\begin{array}{l}\text { Rhamnus } \\
\text { cathartica }\end{array}$ & England & 2006 & HM131247 & HM147299 & - & - \\
\hline BPI 60120 & IV & no & $\begin{array}{l}\text { Unknown } \\
\text { grass }\end{array}$ & Columbia & 1971 & HM131242 & - & - & - \\
\hline
\end{tabular}


Table 1 (continued)

\begin{tabular}{lllllllll}
\hline $\begin{array}{l}\text { Species Name } \\
\text { and Voucher } \\
\text { Number }\end{array}$ & Clade PCA & Country & Year & ITS $^{\mathrm{c}}$ & BT & COI & RPB2 \\
\hline
\end{tabular}

P. coronata var coronata (Clade VII): mainly on Calamagrostis; Europe

\begin{tabular}{|c|c|c|c|c|c|c|c|c|c|}
\hline BR 3933-53 & VII & no & $\begin{array}{l}\text { Agrostis } \\
\text { stolonifera }\end{array}$ & Belgium & 1988 & HM131315 & - & - & - \\
\hline $\begin{array}{l}\text { PRM } 155608 \\
\text { (ET) }\end{array}$ & VII & no & $\begin{array}{r}\text { Calamagrostis } \\
\text { arundinacea }\end{array}$ & Bohemia & $\mathrm{n} / \mathrm{a}$ & HM131309 & - & - & - \\
\hline BP 89076 & VII & yes & $\begin{array}{l}\text { Calamagrostis } \\
\text { epigeios }\end{array}$ & Hungary & 1991 & HM057141 & HM068003 & HM147414 & HM147362 \\
\hline BP 89848 & VII & yes & $\begin{array}{l}\text { Calamagrostis } \\
\text { epigeios }\end{array}$ & Hungary & 1989 & HM131314 & HM147291 & HM147413 & HM147361 \\
\hline $\mathrm{K}(\mathrm{M}): 107200$ & VII & no & $\begin{array}{l}\text { Frangula } \\
\text { almus }\end{array}$ & England & 1989 & HM131316 & - & - & - \\
\hline $\begin{array}{l}\text { (S)reg.nr. } \\
\text { F46266 }\end{array}$ & VII & yes & $\begin{array}{l}\text { Frangula } \\
\text { almus }\end{array}$ & Sweden & 2005 & HM131310 & HM147296 & HM147420 & HM147367 \\
\hline B 700006597 & VII & yes & $\begin{array}{l}\text { Frangula } \\
\text { almus }\end{array}$ & Germany & 2003 & HM131312 & HM147302 & HM147427 & - \\
\hline BP 89353 & VII & no & $\begin{array}{l}\text { Frangula } \\
\text { almus }\end{array}$ & Hungary & 1991 & HM131313 & - & - & HM147360 \\
\hline B 700012416 & VII & no & $\begin{array}{l}\text { Rhamnus } \\
\text { saxatilis }\end{array}$ & Austria & 1995 & HM131311 & - & - & - \\
\hline
\end{tabular}

P. coronati-agrostis Liu \& Hambleton sp. nov. (Clade IX): on Agrostis and Phalaris; Europe, North America

\begin{tabular}{|c|c|c|c|c|c|c|c|c|c|}
\hline $\mathrm{K}(\mathrm{M}): 82517$ & IX & yes & $\begin{array}{l}\text { Agrostis cf } \\
\text { tenuis }\end{array}$ & England & 2000 & HM131323 & HM147300 & - & HM147371 \\
\hline PUR 54591 & IX & no & $\begin{array}{l}\text { Agrostis } \\
\text { gigantea }\end{array}$ & USA & 1955 & HM131324 & - & - & - \\
\hline PUR 55024 & IX & no & $\begin{array}{l}\text { Agrostis } \\
\text { gigantea }\end{array}$ & USA & 1955 & HM131325 & HM147328 & - & - \\
\hline PUR 59741 & IX & no & $\begin{array}{l}\text { Agrostis } \\
\text { gigantea }\end{array}$ & Canada & 1960 & HM131326 & & - & - \\
\hline PUR N1029 & IX & no & Agrostis sp. & Canada & 1994 & HM131327 & HM147287 & HM147406 & - \\
\hline BR 392848 & IX & no & $\begin{array}{l}\text { Agrostis } \\
\text { stolonifera }\end{array}$ & Belgium & 1988 & HM131320 & - & - & - \\
\hline PUR N114 (T) & IX & yes & $\begin{array}{l}\text { Agrostis } \\
\text { stolonifera }\end{array}$ & Finland & 1977 & HM131319 & - & HM147393 & HM147379 \\
\hline PUR N5372 & IX & yes & $\begin{array}{l}\text { Agrostis } \\
\text { stolonifera }\end{array}$ & USA & 1992 & HM131329 & - & HM147407 & HM147355 \\
\hline K(M) 60529 & IX & yes & $\begin{array}{l}\text { Agrostis } \\
\text { stolonifera }\end{array}$ & Austria & 1994 & HM131322 & - & HM147425 & HM147372 \\
\hline PUR N1268 & IX & no & $\begin{array}{l}\text { Phalaris } \\
\text { arundinacea }\end{array}$ & Canada & 1994 & HM131328 & - & & - \\
\hline DAOM 220642 & IX & yes & Rhamnus sp. & Canada & 1995 & HM131321 & - & HM147397 & HM147345 \\
\hline DAOM 240722 & IX & no & $\begin{array}{l}\text { Rhamnus } \\
\text { cathartica }\end{array}$ & Canada & 2006 & - & HM147340 & HM147401 & - \\
\hline
\end{tabular}

P. coronati-brevispora Liu \& Hambleton sp. nov. (Clade III): on Bromus inermis; North America

\begin{tabular}{|c|c|c|c|c|c|c|c|c|c|}
\hline DAOM 240063 & III & yes & Bromus inermis & Canada & 2006 & HM057139 & HM068001 & HM147404 & HM147353 \\
\hline DAOM 240183 & III & yes & Bromus inermis & Canada & 2006 & HM131237 & HM147289 & HM147412 & HM147359 \\
\hline PUR N1371 & III & yes & Bromus inermis & USA & 1997 & HM131238 & HM147322 & HM147387 & HM147377 \\
\hline PUR N652 (T) & III & yes & $\begin{array}{l}\text { Bromus } \\
\quad \text { inermis }\end{array}$ & USA & 1999 & HM131235 & HM147321 & - & - \\
\hline DAOM 235159 & III & yes & $\begin{array}{l}\text { Rhamnus } \\
\text { cathartica }\end{array}$ & Canada & 2005 & HM131236 & HM147315 & HM147382 & - \\
\hline
\end{tabular}

P. coronati-calamagrostidis Liu \& Hambleton nom. et stat nov. (Clade VI): mainly on Calamagrostis canadensis; North America

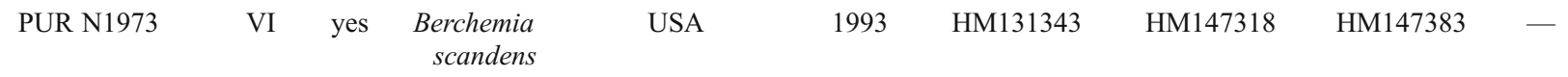


Table 1 (continued)

\begin{tabular}{lllllllll}
\hline $\begin{array}{l}\text { Species Name } \\
\text { and Voucher } \\
\text { Number }\end{array}$ & Clade PCA & Country & Year & ITS $^{\mathrm{c}}$ & BT & COI & RPB2 \\
\hline
\end{tabular}

\begin{tabular}{|c|c|c|c|c|c|c|c|c|c|}
\hline BPI 59821 & VI & no & $\begin{array}{l}\text { Bromus } \\
\text { anomalus }\end{array}$ & USA & 1962 & HM131341 & - & - & - \\
\hline PUR 57282 & VI & no & $\begin{array}{l}\text { Bromus } \\
\text { anomalus }\end{array}$ & USA & 1960 & HM131351 & - & - & - \\
\hline DAOM 204923 & VI & yes & $\begin{array}{l}\text { Bromus } \\
\text { ciliatus }\end{array}$ & Canada & 1918 & HM131305 & HM147339 & - & - \\
\hline DAOM 186149 & VI & no & $\begin{array}{l}\text { Bromus } \\
\quad \text { pumpellianus }\end{array}$ & Canada & 1980 & HM131348 & - & - & - \\
\hline BPI 747648 & VI & yes & $\begin{array}{l}\text { Calamagrostis } \\
\text { canadensis }\end{array}$ & USA & 1995 & HM131303 & - & HM147430 & HM147375 \\
\hline DAOM 107653 & VI & yes & $\begin{array}{c}\text { Calamagrostis } \\
\text { canadensis }\end{array}$ & USA & 1964 & HM131304 & HM147337 & HM147400 & HM147347 \\
\hline $\begin{array}{l}\text { PUR } 22155 \\
\text { (LT) }\end{array}$ & VI & no & $\begin{array}{c}\text { Calamagrostis } \\
\text { canadensis }\end{array}$ & Canada & 1917 & HM131302 & - & - & - \\
\hline PUR 22167 & VI & no & $\begin{array}{c}\text { Calamagrostis } \\
\text { canadensis }\end{array}$ & Canada & 1917 & HM131306 & - & - & - \\
\hline PUR 22185 & VI & no & $\begin{array}{c}\text { Calamagrostis } \\
\text { canadensis }\end{array}$ & USA & 1915 & HM131307 & - & - & - \\
\hline PUR N2268 & VI & yes & $\begin{array}{l}\text { Calamagrostis } \\
\text { canadensis }\end{array}$ & USA & 2000 & HM131308 & HM147320 & HM147385 & - \\
\hline PUR 66551 & VI & no & $\begin{array}{l}\text { Calamagrostis } \\
\text { rubescens }\end{array}$ & USA & 1982 & HM131352 & HM147335 & - & - \\
\hline DAOM 195783 & VI & no & $\begin{array}{l}\text { Elaeagnus } \\
\text { commutata }\end{array}$ & Canada & 1960 & HM131349 & - & - & - \\
\hline DAOM 220889 & VI & yes & Elymus sp. & Canada & 1996 & HM131350 & HM147336 & HM147398 & - \\
\hline BPI 1100292 & VI & no & $\begin{array}{l}\text { Leymus } \\
\text { innovatus }\end{array}$ & Canada & 1953 & HM131346 & - & - & - \\
\hline DAOM 130314 & VI & no & $\begin{array}{l}\text { Shepherdia } \\
\text { canadensis }\end{array}$ & Canada & 1969 & HM131347 & - & HM147396 & - \\
\hline PUR 54152 & VI & no & $\begin{array}{l}\text { Trisetum } \\
\text { canescens }\end{array}$ & USA & 1934 & HM131342 & - & - & - \\
\hline \multicolumn{10}{|c|}{ P. coronati-hordei Liu \& Hambleton nom. et stat. nov. (Clade I): on Elymus and Hordeum; North America } \\
\hline PUR N1396 & I & no & Elymus hystrix & USA & 1996 & HM131227 & 一 & - & - \\
\hline DAOM 183691 & I & yes & $\begin{array}{l}\text { Elymus repens } \\
\text { (= Agropyron } \\
\text { repens) }\end{array}$ & Canada & 1982 & HM057138 & HM068000 & HM147399 & HM147346 \\
\hline PUR N1406 & I & no & $\begin{array}{l}\text { Elymus repens } \\
\text { (= Agropyron } \\
\text { repens) }\end{array}$ & USA & 1995 & HM131228 & HM147327 & - & - \\
\hline PUR N1413 & I & no & $\begin{array}{l}\text { Elymus repens } \\
\text { (= Agropyron } \\
\text { repens) }\end{array}$ & USA & 1992 & HM131229 & - & - & - \\
\hline PUR N1426 & I & yes & $\begin{array}{l}\text { Elymus repens } \\
\text { (= Agropyron } \\
\text { repens) }\end{array}$ & USA & 1995 & HM131230 & HM147334 & - & - \\
\hline PUR N1358 & I & no & $\begin{array}{l}\text { Elymus } \\
\text { virginicus }\end{array}$ & USA & 1995 & HM131226 & HM147326 & - & - \\
\hline PUR N1539 & I & no & $\begin{array}{l}\text { Hordeum } \\
\text { jubatum }\end{array}$ & USA & 1992 & HM131231 & - & - & - \\
\hline PUR 89857 (T) & I & yes & $\begin{array}{l}\text { Hordeum } \\
\text { vulgare }\end{array}$ & USA & 1992 & HM131225 & - & - & - \\
\hline
\end{tabular}

P. coronati-japonica Liu \& Hambleton sp. nov. (Clade VIII): on Calamagrostis arundinacea; Japan

\begin{tabular}{|c|c|c|c|c|c|c|c|c|c|}
\hline PUR N1055 & VIII & yes & $\begin{array}{l}\text { Berchemia } \\
\text { pauciflora }\end{array}$ & Japan & 1990 & HM131318 & HM147319 & HM147384 & - \\
\hline $\begin{array}{l}\text { PUR F16131 } \\
\text { (T) }\end{array}$ & VIII & yes & $\begin{array}{r}\text { Calamagrostis } \\
\text { arundinacea }\end{array}$ & Japan & 1958 & HM131317 & HM147331 & HM147391 & HM147378 \\
\hline
\end{tabular}


Table 1 (continued)

\begin{tabular}{lllllllll}
\hline $\begin{array}{l}\text { Species Name } \\
\text { and Voucher } \\
\text { Number }\end{array}$ & Clade PCA $^{\mathrm{b}}$ Host & Country & Year & ITS $^{\mathrm{c}}$ & BT & COI & RPB2 \\
\hline
\end{tabular}

P. coronati-longispora Liu \& Hambleton sp. nov. (Clade II): on Bromus erectus; Europe

\begin{tabular}{|c|c|c|c|c|c|c|c|}
\hline PRC 194 & II & yes & $\begin{array}{l}\text { Bromus } \\
\text { erectus }\end{array}$ & Slovakia & 2002 & HM131233 & HM147338 \\
\hline PRC 196 (T) & II & no & $\begin{array}{l}\text { Bromus } \\
\text { erectus }\end{array}$ & $\begin{array}{l}\text { Czech } \\
\text { Republic }\end{array}$ & 2002 & HM131232 & - \\
\hline PRC 194 & II & no & $\begin{array}{l}\text { Rhamnus } \\
\text { saxatilis }\end{array}$ & Slovakia & 2002 & HM131234 & - \\
\hline PRC 247 & II & yes & $\begin{array}{l}\text { Rhamnus } \\
\text { saxatilis }\end{array}$ & Slovakia & 2002 & HM057142 & HМ068004 \\
\hline
\end{tabular}

P. coronata s.l. var. gibberosa (Lagerh.) Joerst. (Type seen but not sequenced)

$\begin{array}{lccccccc}\begin{array}{l}\text { BRNM 111952 } \\ \text { (T of var. }\end{array} & \text { XI } & \text { no } & \begin{array}{c}\text { Calamagrostis } \\ \text { epigejos }\end{array} & \begin{array}{c}\text { Czech } \\ \text { Republic }\end{array} & 1923 & \text { HM131332 } & \text { - } \\ \begin{array}{l}\text { intermedia }) \\ \text { PUR F17485 }\end{array} & \text { XI } & \text { no } & \begin{array}{c}\text { Festuca } \\ \text { altissima }\end{array} & \text { Slovakia } & 1960 & \text { HM131333 } & \text { - }\end{array}$

P. coronata s.l. var. himalensis Barclay (Type not seen)

\begin{tabular}{|c|c|c|c|c|c|c|}
\hline $\begin{array}{l}\text { PUR F15640 } \\
\text { (ST of } P . \\
\text { brevicornis) }\end{array}$ & $\mathrm{X}$ & no & $\begin{array}{c}\text { Calamagrostis } \\
\text { canadensis }\end{array}$ & Japan & 1907 & HM131330 \\
\hline $\begin{array}{l}\text { PUR F15641 } \\
\text { (ST of } P \text {. }\end{array}$ & $\mathrm{X}$ & yes & $\begin{array}{c}\text { Calamagrostis } \\
\text { canadensis }\end{array}$ & Japan & 1907 & HM131331 \\
\hline
\end{tabular}

brevicornis)

P. coronata s.1.

BPI 746001

HMAS 65723
BPI 718354
(basal to III)
PUR 25955
(T of $P$.
subdigitata)
PUR N5403

PUR N5403

PUR 59504

PUR N1057

PUR F4486

(T of $P$.

melicae)

PUR N1983

\begin{tabular}{|c|c|c|}
\hline XII & no & $\begin{array}{l}\text { Rhamnus } \\
\text { schneideri }\end{array}$ \\
\hline XIII & yes & $\begin{array}{l}\text { Pennisetum } \\
\text { flaucidum? }\end{array}$ \\
\hline XIV & no & Bromus erectus \\
\hline XV & no & $\begin{array}{l}\text { Brachypodium } \\
\text { mexicanum }\end{array}$ \\
\hline XVI & no & $\begin{array}{l}\text { Rhamnus } \\
\text { japonica }\end{array}$ \\
\hline XVII & no & $\begin{array}{l}\text { Brachypodium } \\
\text { mexicanum }\end{array}$ \\
\hline XVIII & yes & $\begin{array}{l}\text { Helictotrichon } \\
\text { virescens }\end{array}$ \\
\hline XIX & no & Melica nutans \\
\hline XX & no & $\begin{array}{l}\text { Rhamnus } \\
\text { alnifolia }\end{array}$ \\
\hline
\end{tabular}

Chin

China

1985

Germany

1990

HM131334

Germany

1949

Guatemala

1915

Japan

1973

Mexico

1961

Nepal

1986

HM131338

Nepal

Sweden

1894

USA

1994

HM131339

-

HM

HM131345

HM131336

Outgroup Taxa

P. brachypodii

DAOM 185200

P. bromina

BPI 70086

P. cesatii

DAOM 214752

P. durangensis

DAOM 163064

$\begin{array}{cl}\text { no } & \begin{array}{c}\text { Brachypodium } \\ \text { silvaticum } \\ \text { Bromus rubens }\end{array} \\ \text { no } & \begin{array}{c}\text { Andropogon } \\ \text { ischaemum }\end{array} \\ \text { no } & \begin{array}{c}\text { Stipa pringlei } \\ \text { yes }\end{array} \\ \text { Elymus repens }\end{array}$

Hungary

1980

USA

1941

Hungary

1991

USA

1975

Belgium

2001

HM131353

HM131354

HM131355

HM131356

HM131357

HM147308 $\begin{array}{ll}\text { HM147435 } & - \\ \text { HM147408 } & \text { HM147356 }\end{array}$

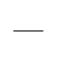

-

HM147395 HM147381

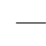

HM147350 
Table 1 (continued)

\begin{tabular}{|c|c|c|c|c|c|c|c|c|c|}
\hline $\begin{array}{l}\text { Species Name } \\
\text { and Voucher } \\
\text { Number }^{\mathrm{a}}\end{array}$ & Clade & $\mathrm{PCA}^{\mathrm{b}}$ & Host & Country & Year & $\mathrm{ITS}^{\mathrm{c}}$ & BT & COI & RPB2 \\
\hline \multicolumn{10}{|l|}{$\begin{array}{l}\text { P. graminis } \\
\text { BR 150069-10 }\end{array}$} \\
\hline $\begin{array}{l}\text { P. graminis } \\
\text { PUR } 66554\end{array}$ & & no & $\begin{array}{l}\text { Oryzopsis } \\
\text { exigua }\end{array}$ & USA & 1982 & HM131358 & - & - & - \\
\hline $\begin{array}{l}\text { P. graminis } \\
\text { BPI } 803290\end{array}$ & & no & $\begin{array}{l}\text { Elytrigia } \\
\text { repens }\end{array}$ & China & 1986 & HM131359 & - & - & - \\
\hline $\begin{array}{l}\text { P. poae- } \\
\text { nemoralis } \\
\text { DAOM } 212041\end{array}$ & & no & Poa pratensis & Canada & 1990 & HM057152 & - & - & - \\
\hline $\begin{array}{l}\text { P. poae- } \\
\text { nemoralis } \\
\text { DAOM } 189681\end{array}$ & & no & $\begin{array}{l}\text { Arctagrostis } \\
\text { latifolia }\end{array}$ & Canada & 1980 & HM057153 & - & - & - \\
\hline $\begin{array}{l}\text { P. poarum } \\
\text { DAOM } 193284\end{array}$ & & no & Poa pratensis & Canada & 1983 & HМ057149 & - & - & - \\
\hline $\begin{array}{l}\text { P. poarum } \\
\text { DAOM } 240188\end{array}$ & & no & $\begin{array}{l}\text { Tussilago } \\
\text { farfara }\end{array}$ & Canada & 2006 & HM057150 & - & - & - \\
\hline $\begin{array}{l}\text { P. recondita } \\
\text { DAOM } 192559\end{array}$ & & no & $\begin{array}{l}\text { Triticum } \\
\text { aestivum }\end{array}$ & Australia & 1982 & HM057145 & - & - & - \\
\hline $\begin{array}{l}\text { P. recondita } \\
\text { DAOM } 240185\end{array}$ & & no & $\begin{array}{l}\text { Agropyron } \\
\text { repens }\end{array}$ & Canada & 2006 & HM131360 & HM147309 & - & - \\
\hline $\begin{array}{l}\text { P. striiformis } \\
\text { PUR N5378 }\end{array}$ & & no & $\begin{array}{l}\text { Dactylis } \\
\text { glomerata }\end{array}$ & USA & 1992 & HМ057109 & - & - & - \\
\hline $\begin{array}{l}\text { P. striiformis } \\
\text { DAOM } 240071\end{array}$ & & yes & $\begin{array}{l}\text { Triticum } \\
\text { aestivum }\end{array}$ & China & 2006 & HМ057121 & HМ067991 & - & HM147369 \\
\hline $\begin{array}{l}\text { P. triticina } \\
\text { BR 59352-85 }\end{array}$ & & no & $\begin{array}{l}\text { Elymus } \\
\text { repens }\end{array}$ & Belgium & 1996 & HM131361 & HM147311 & - & - \\
\hline $\begin{array}{l}\text { P. triticina } \\
\text { B } 700012410\end{array}$ & & no & $\begin{array}{l}\text { Elymus } \\
\text { caninus }\end{array}$ & Romania & 1974 & HM131362 & HM147312 & - & - \\
\hline $\begin{array}{l}\text { Uromyces } \\
\text { alopercuri } \\
\text { DAOM } 234714\end{array}$ & & no & $\begin{array}{l}\text { Alopecurus } \\
\text { aristulatus }\end{array}$ & Canada & 1917 & HM131363 & - & - & - \\
\hline $\begin{array}{l}\text { Uromyces } \\
\text { clignyi } \\
\text { DAOM } 192217\end{array}$ & & no & Andropogon sp. & N. Rhodesia & 1962 & HM131364 & - & - & - \\
\hline $\begin{array}{l}\text { Uromyces } \\
\text { coronatus } \\
\text { DAOM } 32991\end{array}$ & & no & $\begin{array}{l}\text { Zizania } \\
\quad \text { latifolia }\end{array}$ & Japan & 1949 & HM131365 & - & - & - \\
\hline $\begin{array}{l}\text { Uromyces } \\
\text { dactylis } \\
\text { DAOM } 216236\end{array}$ & & yes & $\begin{array}{l}\text { Dactylis } \\
\text { glomerata }\end{array}$ & Hungary & 1992 & HM057148 & HM068010 & - & - \\
\hline
\end{tabular}

${ }^{\mathrm{a}} \mathrm{T}=$ type, ET=epitype, $\mathrm{LT}=$ lectotype, ST=Syntype; B: Botanischer Garten und Botanisches Museum Berlin-Dahlem, Zentraleinrichtung der Freien Universität Berlin, BERLIN, Germany; BP: Hungarian Natural History Museum, BUDAPEST, Hungary; BPI: US National Fungus Collections, BELTSVILLE, Maryland, USA; BR: National Botanic Garden of Belgium, MEISE, Belgium; BRNM: Moravian Museum, BRNO, Czech Republic; DAOM: Agriculture and Agri-Food Canada, OTTAWA, Ontario, Canada; HMAS: Institute of Microbiology, Academia Sinica, BEIJING, People's Republic of China; K(M): Royal Botanic Gardens, KEW, UK, England; PRC: Charles University in Prague, PRAHA, Czech Republic; PRM: National Museum, PRAHA, Czech Republic; PUR: Purdue University, WEST LAFAYETTE, Indiana, USA; S: Swedish Museum of Natural History, STOCKHOLM, Sweden.

${ }^{\mathrm{b}} \mathrm{PCA}=$ presence in combined analysis; yes=present, no=absent.

${ }^{\mathrm{c}}$ Sequences in normal font include ITS2 region only, in bold font include complete ITS region.

Bayesian analysis was conducted for the combined data set to estimate the posterior probability for the branches using MrBayes 3.1 (Huelsenbeck and Ronquist 2001). Each gene partition was defined with different DNA substitution models, estimated with Modeltest 3.6 (Posada and Crandall
1998). Four chains of 5,000,000 Markov chain Monte Carlo generations were run. The first 5000 trees were discarded (burn-in), and the remaining trees were spooled to PAUP* to obtain posterior probabilities based on the $50 \%$ majorityrule consensus. 
Morphological examination

Dried herbarium specimens were softened in a moist chamber for at least $30 \mathrm{~min}$. Entire fungal sori or clumps of spores were removed using tweezers and mounted on microscope slides in lactic acid cotton blue media. To observe germ pores, clumps of urediniospores were mounted in Congo Red (Urban 1963). All cross-sections were cut using a MicroTome Crystat, model HM500 OMs (MICROM Laborgeräte GmbH, Walldorf, Germany). Slide mounts were examined using an Olympus BX51 Differential Interference Contrast Light Microscope (Olympus Canada Inc., Markham, Canada). Macroscopic morphology was examined using a Zeiss Discovery v12 Stereomicrosope (Carl Zeiss MicroImaging GmbH, Germany). Colors of sori were recorded using Kornerup and Wanscher (1967). Digital micrographs were taken with an Olympus DP 70 camera and analyzed by Image-Pro Plus ver. 6.0 Image Processing and Analysis Software (MediaCybernetics, Inc. Bethesda, USA) to obtain measurements.

The morphological characters examined in this study were mainly from uredinial and telial stages because specimens with aecial sori were not well-represented for each clade in our analyses. With the goal of finding synapomorphies supporting the main lineages, the following characters were examined: telia/uredinia - location of sori (abaxial/ adaxial), color, size, openness (exposed/covered), and degree of loculation; telial paraphyses - presence/absence and shape (cylindrical/fused); teliospores - shape (clavate/ obvoid, constricted at septum or not), size (length of spores, length of lower cell, width of widest spot of upper cell), apical wall color and thickness, digitations (number and length of the longest digitation), pedicles (length of the remnant and width of hilum); urediniospores - size, surface ornamentation (distance between warts), germ pores (number and invagination). Selected quantitative characters, including teliospore length/width, width of teliospore hilum, length of longest digitations per teliospore, urediniospore length and width, were analyzed using Student's $t$-test. Pair-wise comparisons of means for each character, calculated for taxa within
Fig. 1 Phylogenetic relationships of 154 OTUs inferred from parsimony analyses based on the ITS region. $\mathrm{L}=720, \mathrm{CI}=0.524, \mathrm{RI}=0.836$, $\mathrm{RC}=0.438, \mathrm{HI}=0.476$, and $\mathrm{G}-\mathrm{fit}=-138.126$. Bootstrap values higher than $50 \%$ are shown on the corresponding branches. Bold font indicates an OTU included in the combined analysis (Fig. 3). The bars corresponding to Clades I-XI are colour-coded to match the taxonomic groups shown in Fig. 3. Sequence labels include specimen accession number, species name $(\mathrm{P} .=$ Puccinia, $\mathrm{Pc}=$ Puccinia coronata $)$, host name, source. Those ending in clade designations XII-XIX (red font) indicate de novo lineages, $\dagger$ indicates a new type designated in this study, indicates the seven previously designated types listed in the Phylogentic Analyses section, Results and discussion

each clade, were performed to assess whether they were statistically different among clades.

\section{Results and discussion}

Genomic DNA extraction, PCR amplification and sequencing

Herbarium specimens of $P$. coronata sensu lato (s.1.) were successfully amplified and sequenced for the ITS region more often than for the protein-coding gene regions. For recently collected specimens, i.e. after the 1990s, the amplification success rate was $74 \%$ while for those collected earlier than 1980, it decreased by about half to $34 \%$ (Table 2). In total, 147 of $291(50.5 \%)$ processed specimens were successfully sequenced for the complete ITS region or for ITS2 alone (340$500 \mathrm{nt}$ ), of which 133 were selected for the analyses.

For the protein-coding genes, samples successfully sequenced were 54 of 64 attempted for COI (330 nt), 55 of 257 for BT (500-800 nt) and 32 of 257 for RPB2 (270-500 nt). Among 73 DNA samples attempted for PreCR repair, at least 30 were effectively amplified for at least one gene region. Overall older specimens were more difficult to amplify successfully. PrePCR treatment helped in some cases but success was unpredictable. For instance, the treatment was successful for the type specimen of $P$. coronata collected in 1894 but also for some recently collected specimens and not other older

Table 2 Number of DNA extractions and rDNA sequences obtained from P. coronata herbarium specimens

\begin{tabular}{|c|c|c|c|c|c|c|c|c|c|c|c|c|c|c|}
\hline $\begin{array}{l}\text { Year } \\
\text { specimen } \\
\text { collected }\end{array}$ & $>2005$ & $\begin{array}{l}2004- \\
2000\end{array}$ & $\begin{array}{l}1999- \\
1995\end{array}$ & $\begin{array}{l}1994- \\
1990\end{array}$ & $\begin{array}{l}1989- \\
1985\end{array}$ & $\begin{array}{l}1984- \\
1980\end{array}$ & $\begin{array}{l}1979- \\
1975\end{array}$ & $\begin{array}{l}1974- \\
1970\end{array}$ & $\begin{array}{l}1969- \\
1965\end{array}$ & $\begin{array}{l}1964- \\
1960\end{array}$ & $\begin{array}{l}1959- \\
1955\end{array}$ & $\begin{array}{l}1954- \\
1950\end{array}$ & $<1949$ & Total \\
\hline $\begin{array}{l}\text { Number of } \\
\text { DNA } \\
\text { extractions }\end{array}$ & 38 & 24 & 29 & 31 & 20 & 25 & 12 & 15 & 10 & 29 & 20 & 10 & 28 & 291 \\
\hline $\begin{array}{l}\text { Number of } \\
\text { obtained } \\
\text { ITS } \\
\text { sequences }\end{array}$ & 28 & 18 & 19 & 24 & 11 & 10 & 3 & 5 & 1 & 9 & 4 & 1 & 14 & 147 \\
\hline Rates (\%) & 73.7 & 75 & 65.5 & 77.4 & 55 & 40 & 25 & 33.3 & 10 & 31.0 & 20 & 10 & 50 & 50.5 \\
\hline
\end{tabular}




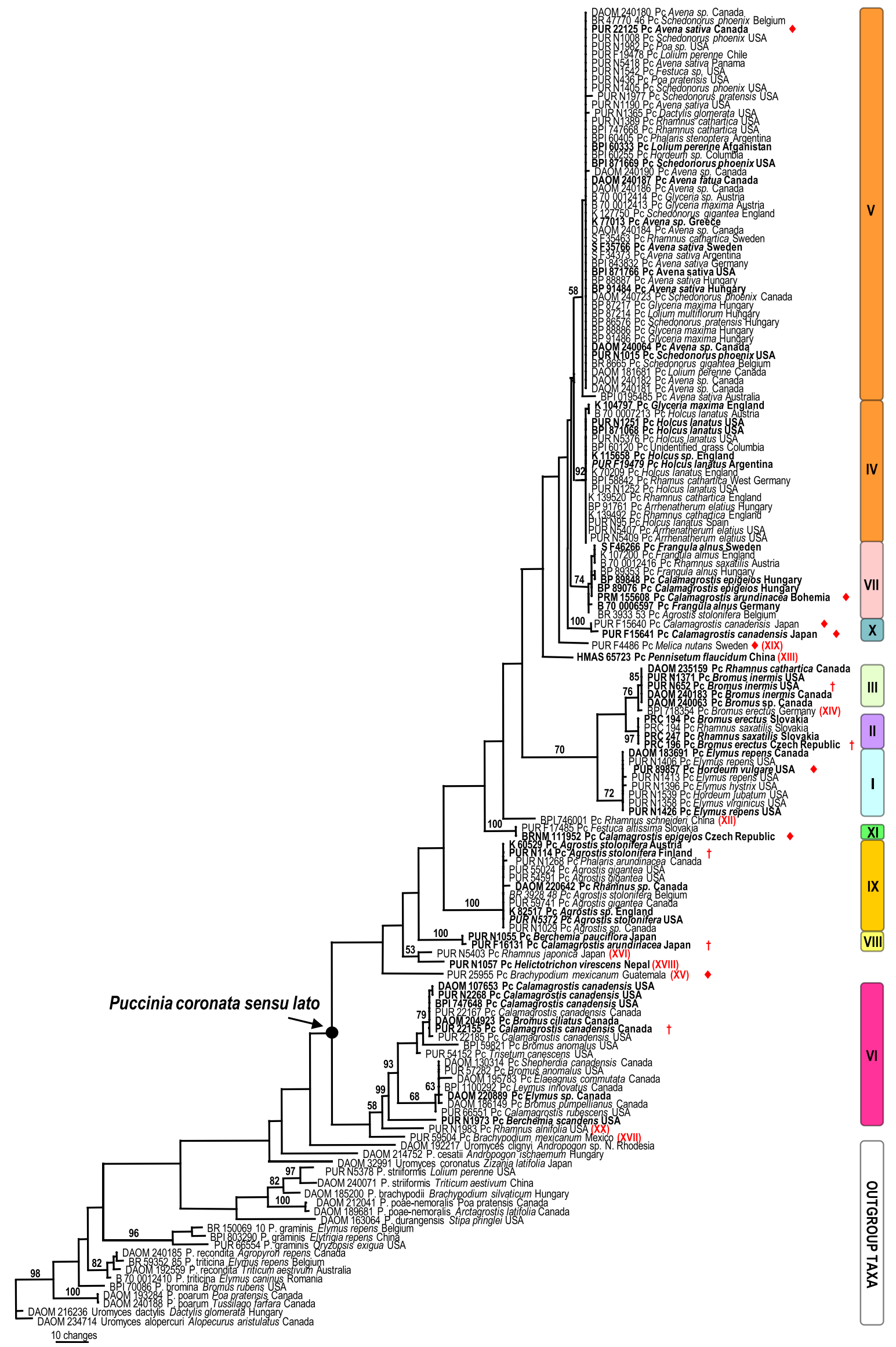


a

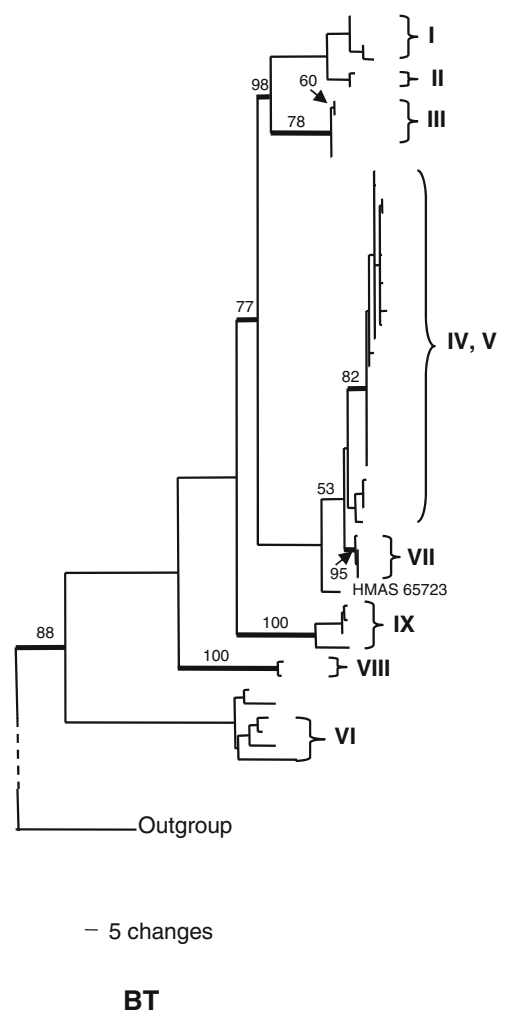

b

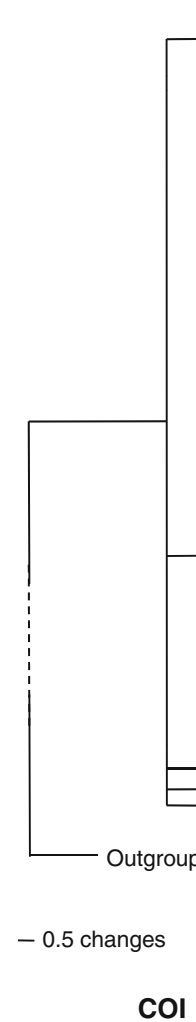

C

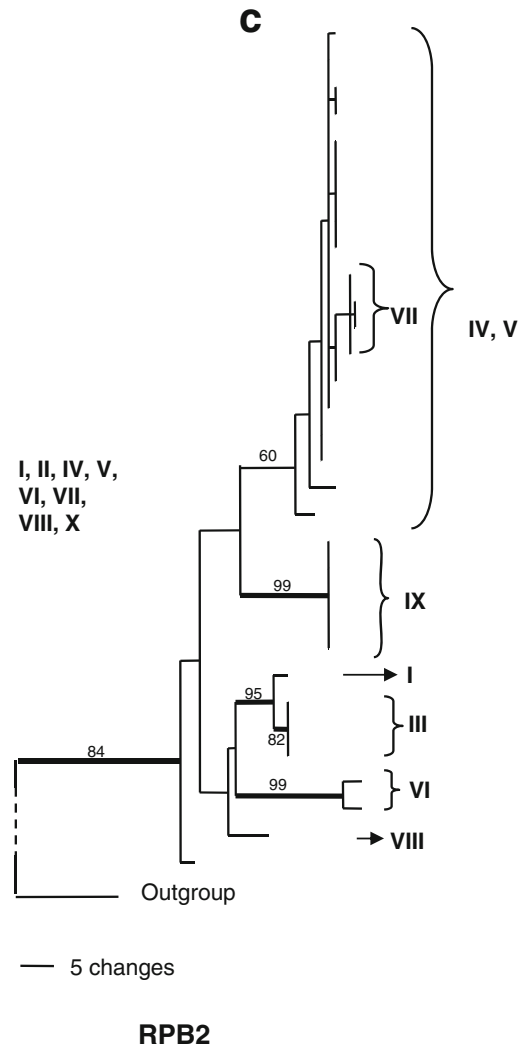

Fig. 2 Phylogenetic lineages of Puccinia coronata s.l. recovered by protein coding genes. a. BT, 1 of 65975 most parsimonious trees (MPTs), parsimony informative characters $=250, \mathrm{~L}=736, \mathrm{CI}=0.662$, $\mathrm{RI}=0.856, \mathrm{RC}=0.567, \mathrm{HI}=0.338$, and $\mathrm{G}-\mathrm{fit}=-196.036$; $\mathbf{b}$ : COI, 1 of

specimens. The oldest specimen amplified and sequenced without PreCR treatment was collected in 1907.

\section{Phylogenetic analyses}

Partitioned analyses were conducted for each gene individually because of the disparity in taxon sets. Clade designations were applied as consistently as possible in the descriptions of the phylogenetic hypotheses resulting from each analysis.

ITS The data matrix comprised 133 P. coronata, 21 outgroup taxa and 734 characters (with insertions and deletions) of which 190 were parsimony informative. Parsimony analysis recovered 18,231 most parsimonious trees with length $(\mathrm{L})=720, \mathrm{CI}=0.524, \mathrm{RI}=0.836, \mathrm{RC}=$ $0.438, \mathrm{HI}=0.476$, and $\mathrm{G}-\mathrm{fit}=-138.126$. Eleven major clades with moderate to high bootstrap support were recognized as shown on Fig. 1 (I-XI) plus nine additional single taxon clades (XII-XX). Six of these (Clade I-VI) were congruent with results from the previous study by Szabo (2006). For convenience, these clades were similarly labelled as Clades I-VI on Fig. 1. Consequently, the additional five new major clades were labelled as VII-XI.
182,815 MPTs, parsimony informative characters $=23, \mathrm{~L}=52, \mathrm{CI}=$ $0.962, \mathrm{RI}=0.974, \mathrm{RC}=0.936, \mathrm{HI}=0.038, \mathrm{G}-\mathrm{fit}=-22.500$; $\mathbf{c}: \mathrm{RPB} 2,1$ of 14476 MPTs, parsimony informative characters $=145, \mathrm{~L}=371, \mathrm{CI}=$ $0.728, \mathrm{RI}=0.796, \mathrm{RC}=0.579, \mathrm{HI}=0.272, \mathrm{G}-\mathrm{fit}=-123.357$

The nine single taxon clades potentially represented de novo lineages within the $P$. coronata complex: BPI 746001 (XII) and HMAS 65723 (XIII) from China, BPI 718354 (XIV) from Germany (sister to the North American Clade III, see Discussion), PUR 25955 (XV) from Guatemala (type of $P$. subdigitata), PUR N5403 (XVI) from Japan, PUR 59504 (XVII) from Mexico, PUR N1057 (XVIII) from Nepal, PUR F4486 (XIX) from Sweden (type of P. melicae), and PUR N1983 (XX) from USA. ITS sequences were lacking for only two collections sampled for this study: DAOM 240722 from Canada which groups with Clade IX based on BT data only (not included in the final analyses) and PUR F16064 from Great Britain which groups with Clade IV/V based on RPB2 data but Clade IV based on host association (data not shown).

Seven previously designated types were included in the ITS analysis. The phylogenetic placement of these reference specimens in the ITS phylogram (Fig. 1; indicated by the symbol $\downarrow$ in red font) was as follows. Clade V: type of $P$. coronata var. avenae Fraser \& Ledingham (PUR 22125, lectotypified by Cummins [1971]); Clade VII: type of $P$. coronata var. coronata Corda (PRM 155608); Clade X: $P$. brevicornis Ito (PUR F15640 and PUR F15641, syntypes), synonym of $P$. coronata var himalensis (Barcl.) Cummins 1971; Clade I: type of P. coronata var. hordei Jin and 


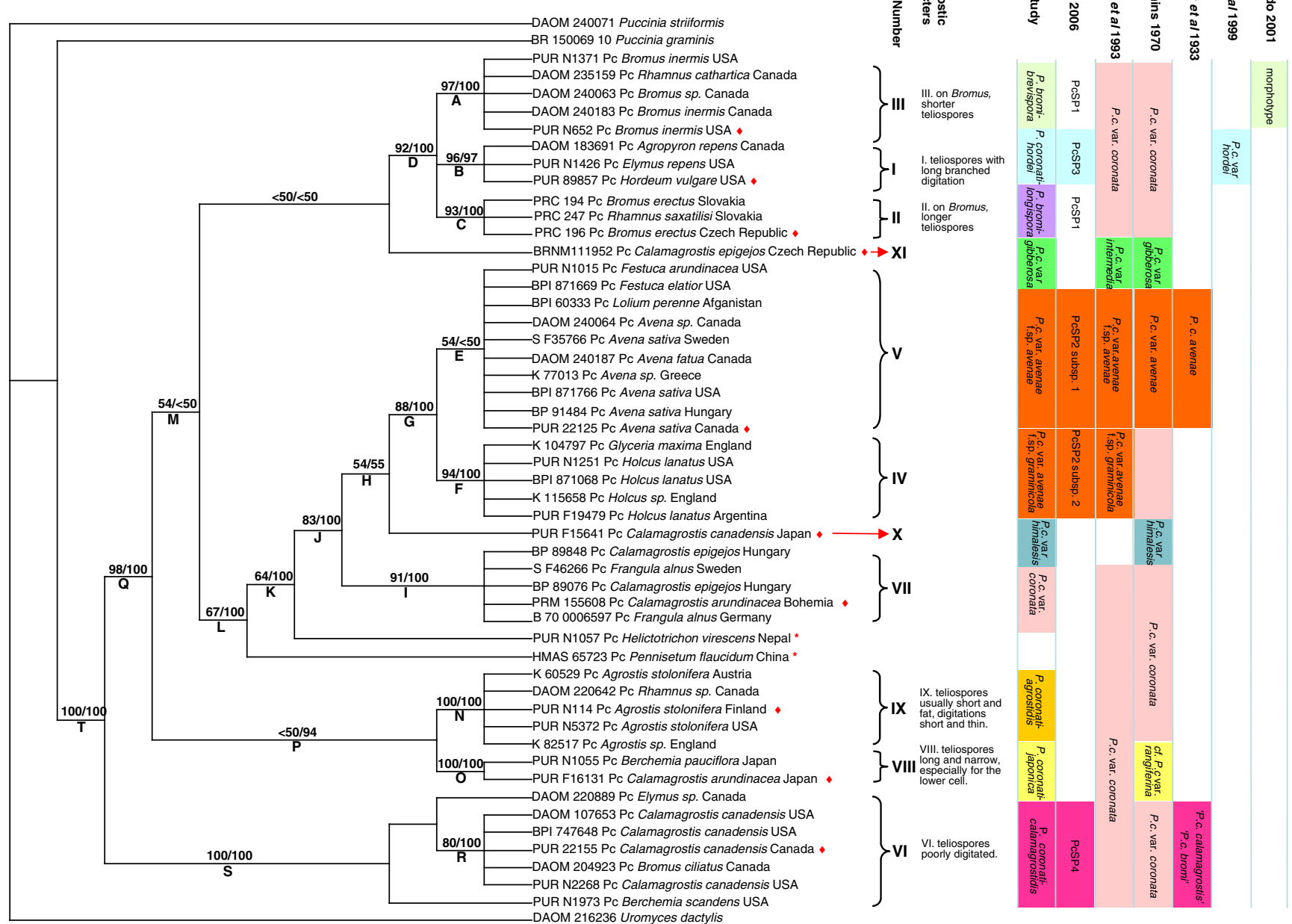

Fig. 3 Strict consensus of 60,916 most parsimonious trees based on ITS, BT, COI and RPB2 combined data set. Parsimony informative characters $=370, \mathrm{~L}=1149, \mathrm{CI}=0.746, \mathrm{RI}=0.828, \mathrm{RC}=0.617, \mathrm{HI}=$ 0.254 , G-fit $=-308.314$. Bootstrap values/posterior probability values are shown on the corresponding branches. Major nodes labeled as A-T were analyzed for Partitioned Bremer Support (see Table 3). In co-

Steffenson 1999 (PUR 89857); Clade XI: type of P. coronata var. intermedia Urban 1967 (BRNM 111952), accepted as a synonym of $P$. coronata var gibberosa (see Taxonomy section); and two single taxon lineages: type of $P$. melicae Sydow (PUR F4486, Clade XIX, sister to Clade IV+V+VII+ $\mathrm{X}$ ) and type of $P$. subdigitata Arth. \& Holw. (PUR 25955, Clade $\mathrm{XV})$. Types were designated in this study for Clades II, III, VI, VIII and IX (Fig. 1; indicated by the symbol $\uparrow$ in red font).

$B T$ Fifty four $P$. coronata and nine outgroup specimens were included in an alignment of 837 characters, among which 250 characters are parsimony-informative. Parsimony analysis recovered 65975 most parsimonious trees with $\mathrm{L}=$ 736, $\mathrm{CI}=0.662, \mathrm{RI}=0.856, \mathrm{RC}=0.567, \mathrm{HI}=0.338$, and $\mathrm{G}-$ fit $=-196.036$. Clades congruent with the ITS tree include I- lumns to the right of the phylogram, the taxon names accepted here for Clades I-XI are listed in the same colour-coded boxes as in Fig. 1, and compared with previous names or concepts accepted by the researchers indicated at the top of each column. Diagnostic characters are listed for selected taxa (Clades I, II, III, VI, VIII and IX). indicates a type specimen, * indicates a de novo lineage (Clades XIII and XVIII in Fig. 1)

III, VI, VII, VIII, and IX, while an unresolved group comprised Clades IV and V (Fig. 2). DNA sequences for taxa grouping in Clades $\mathrm{X}$ and XI of the ITS tree were not obtained for this locus.

COI Fifty four $P$. coronata and nine outgroup specimens were included in an alignment of 373 characters, among which 23 characters are parsimony informative. Parsimony analysis recovered 182815 most parsimonious trees with $\mathrm{L}=$ 52, $\mathrm{CI}=0.962, \mathrm{RI}=0.974, \mathrm{RC}=0.936, \mathrm{HI}=0.038$, and $\mathrm{G}-$ fit $=-22.500$. The analysis resulted in an unresolved phylogram except for Clade III (Fig. 2).

RPB2 Thirty two P. coronata and seven outgroup specimens were included in an alignment of 501 characters, 
Table 3 Partitioned Bremer Support for selected nodes on combined gene tree

\begin{tabular}{llllll}
\hline Node & ITS & $\beta$-tubulin & COI & RPB2 & Total \\
\hline $\mathrm{A}$ & 1.6 & 2.4 & -0.1 & 0.2 & 4.0 \\
$\mathrm{~B}$ & 1.3 & -0.3 & 0.1 & -0.1 & 1.0 \\
$\mathrm{C}$ & 1.8 & 0.1 & 0.2 & -0.1 & 2.0 \\
$\mathrm{D}$ & 5.5 & 0.2 & -0.5 & -0.2 & 5.0 \\
$\mathrm{E}$ & 3.9 & -2.9 & 0.2 & -0.1 & 1.0 \\
$\mathrm{~F}$ & 1.3 & -0.1 & 0.0 & -0.2 & 1.0 \\
$\mathrm{G}$ & 4.2 & -3.5 & 0.2 & 0.1 & 1.0 \\
$\mathrm{H}$ & 1.6 & -0.7 & 0.2 & -0.1 & 1.0 \\
$\mathrm{I}$ & 0.2 & 2.2 & 0.2 & 0.5 & 3.0 \\
$\mathrm{~J}$ & 0.3 & 0.6 & 0.2 & 0.0 & 1.0 \\
$\mathrm{~K}$ & 0.0 & -2.3 & 0.3 & 4.1 & 2.0 \\
$\mathrm{~L}$ & 0.1 & -2.1 & 0.2 & 3.9 & 2.0 \\
$\mathrm{M}$ & 2.0 & 0.6 & -0.3 & -0.3 & 2.0 \\
$\mathrm{~N}$ & 6.6 & -0.4 & 0.1 & 3.8 & 10.0 \\
$\mathrm{O}$ & 0.0 & 5.6 & 1.2 & 0.2 & 7.0 \\
$\mathrm{P}$ & Not present in strict consensus tree & & \\
$\mathrm{Q}$ & 5.6 & 4.5 & -0.3 & 0.2 & 10.0 \\
$\mathrm{R}$ & 1.6 & 0.3 & 0.0 & 0.2 & 2.0 \\
$\mathrm{~S}$ & 11.6 & 0.6 & -1.4 & 0.2 & 12.0 \\
$\mathrm{~T}$ & 14.5 & -1.3 & 5.6 & 13.2 & 32.0 \\
\hline & & & & &
\end{tabular}

among which 145 characters are parsimony informative. Parsimony analysis recovered 14476 most parsimonious trees with $\mathrm{L}=371, \mathrm{CI}=0.728, \mathrm{RI}=0.796, \mathrm{RC}=0.579, \mathrm{HI}=$ 0.272 , and G-fit $=-123.357$. Clades III, VI, VII and IX were recovered while Clades IV and $\mathrm{V}$ presented as a single unresolved paraphyletic group (Fig. 2). Clades I, VIII and $\mathrm{XI}$ were single-taxon lineages, due to the reduced number of specimens sampled for this gene.

Although there were no conflicts in terms of the groupings of taxa into clades among three loci, ITS, BT and RPB2, relationships among the clades were inconsistent and multiple internal branches received low bootstrap support (BP), likely caused by the limited number of characters. In this case a holistic analysis is more appropriate to maximize the phylogenetic signal (Smith 2000). Partitioned Bremer Support (PBS) was used to measure the degree of support provided by each locus. For the combined data set, we included taxa for which DNA sequences were obtained for at least three loci (with the additional of some type specimens for which only ITS was available). The unavailable loci for some taxa were treated as missing data. Fiftytwo taxa were compiled resulting in a matrix with 2,447 characters, among which 370 characters are parsimony informative. Parsimony analysis recovered 4901 most parsimonious trees with $\mathrm{L}=1149, \mathrm{CI}=0.746, \mathrm{RI}=0.828, \mathrm{RC}=$ $0.617, \mathrm{HI}=0.254$, and G-fit $=-308.493$ (Fig. 3). Nine clades received high $\mathrm{BP}$ support, except Clade $\mathrm{V}$ (branch $\mathrm{E}, \mathrm{BP}=$ $54 \%$ ) and some internal branches $(\mathrm{H}, \mathrm{K}, \mathrm{L}$, and $\mathrm{M})$. The low support for branch $\mathrm{E}$ was predicted by the incongruence between the ITS and BT data sets, as shown by PBS values (ITS $=3.9, \mathrm{BT}=-2.9$, Table 3 ), as well as the low or no support from the other two loci $(\mathrm{COI}=0.2$ and $\mathrm{RPB} 2=$ -0.1). The low support values for branches $\mathrm{K}$ and $\mathrm{L}$ were likely similarly due to the incongruence between $\mathrm{BT}$ and RPB2 (Table 3). In the other cases, signals were generally low while branch $\mathrm{P}$ was not retained in strict consensus tree and thus no PBS was available.

The models and priors for each gene partition estimated using Modeltest were as follows: for ITS, selected model F81 $+\mathrm{I}+\mathrm{G}$, base frequency $(\mathrm{A}, \mathrm{C}, \mathrm{G}, \mathrm{T}) 0.35110 .1889,0.1106$, 0.3494 , all substitution rates equal, proportion of invariable sites $(I)=0.4873$, gamma distribution shape parameter $=$ 0.5811; for BT, $\mathrm{HKY}+\mathrm{G}$ model, base frequency 0.2338 $0.27850 .21040 .2774, \mathrm{ti} / \mathrm{tv}$ ratio $=1.8865, \mathrm{I}=0$, gamma distribution shape parameter $=0.2436$; for COI, $F 81+\mathrm{G}$ model, base frequency 0.25070 .15960 .25450 .3351 , all substitution rates equal, $\mathrm{I}=0$, gamma distribution shape parameter= 0.5346; for RPB2, $\operatorname{TrNef}+\mathrm{I}+\mathrm{G}$, base frequency equal, substitution rates are $1.00004 .7382,1.00001 .00007 .94391 .0000$, $\mathrm{I}=0.3910$, gamma distribution shape parameter $=0.3803$. The Bayesian analyses generally resulted in higher posterior probability (PP) support for most branches (Fig. 3), although branches E, H and M still had low support (both low BP and PP). The tree topology estimated from the combined data set recovered Clade VI as the sister lineage to the one including all other taxa in the $P$. coronata complex. Phylogenetic trees based on ITS, BT, COI, RPB2 and the concatenated matrix can be accessed through TreeBASE at http://purl.org/phylo/ treebase/phylows/study/TB2:S 12078 ? x-accesscode $=$ cda 847 cd 1 fe 4 f973c6106a06f0912e25\&format $=$ html.

\section{Morphology}

Generally speaking, the morphological features useful for characterizing rust fungi are variable within lineages of this species complex and overlapping among them. Student's $t$-test analysis of selected quantitative characters, including teliospore length/width, width of teliospore hilum, length of longest digitations per teliospores, urediniospore length and width, showed that although the ranges overlapped, the means of these measurements were significantly different (Table 4) among some clades and as a result, selected taxa can be recognized using a combination of morphological characters, host association and geographic origin. Groupings of clades based on our statistical tests are summarized in Table 5. A synoptic key is presented to facilitate the identification of the nine accepted taxa (corresponding to Clades I-IX). 
Recognition of taxa

Based on our molecular evidence, in conjunction with morphology, telial host range and geographic distribution, and comparison with the previous classifications, seven species were recognized (Table 1). The type species, $P$. coronata was further divided into two varieties, of which one comprises two formae speciales. All crown rust specimens from oats were resolved as a phylogenetically distinct group within the $P$. coronata complex (for more discussion see $P$. coronata var. avenae, Taxonomy section). Considering the strong support for $P$. coronata s.l. as a monophyletic group (100\%), we erected a new Series Puccinia Series Coronata to define this phylogenetically cohesive lineage. As argued in Liu and Hambleton (2010) for the Series Striiformis, the incorporation of additional subgeneric ranks will contribute to a refined classification within the genus Puccinia.

For each taxon accepted, the host ranges provided are based only on the provenance of the specimens included in the phylogenetic analyses presented here and do not include other hosts documented elsewhere in the literature. Therefore, alternate host associations are listed only if specimens on the aecial host were sampled. Several taxa correspond to previously published concepts for varieties or f. spp. (Fig. 3): two named by Fraser and Ledingham (1933), four by Cummins (1971), four by Urban and Marková (1993), six clades recognized by Szabo (2006); and also the varieties recognized by Jin and Steffenson (1999) and Delgado et al. (2001).

Among members of Series Coronata there is a large degree of genetic diversity, and morphological variation within and between taxa. Multiple orphan taxa were present in our ITS tree, which might represent discrete species, and each one deserves a detailed study based on multiple representatives. Far from being a complete monograph, our review serves as a framework and stepping-stone for further investigation. Although individual clades corresponding to the recognized species were strongly supported by statistical measures, most of the relationships among clades remained unresolved. It is essential to obtain sequences from additional loci and specimens to resolve the uncertain relationships.

\section{Taxonomy}

Puccinia Series Coronata Liu and Hambleton ser. nov. (MycoBank \#: MB563540)

Uredosporis globosis vel ellipsoideis, tenuiter echinulatis, poris $4-15$, sparsis, obscurissimis; teleosporis, bicellularis, vel interdum unicellularis aut tricellularis, clavatis, ad apicum coronatis digitiformis.

Type species: Puccinia coronata A.C.J. Corda

\section{A synoptic key to treated taxa in Puccinia Series Coronata}

Each taxon is referenced in the key by its number assigned as follows:

1. P. coronata var avenae

1a. P. coronata var avenae f. sp. avenae

1b. P. coronata var. avenae f. sp. graminicola

2. P. coronata var. coronata

3. P. coronati-agrostidis

4. P. coronati-brevispora

5. P. coronati-calamagrostidis

6. P. coronati-hordei

7. P. coronati-japonica

8. P. coronati-longispora

Twenty-seven host genera/species were summarized based on examined specimens. For each morphological character there are multiple states. Each character state and host genus/species is connected to numbers, which represent correlated taxa. Underlined numbers indicate taxa exhibiting multiple possible character states.

1. Host

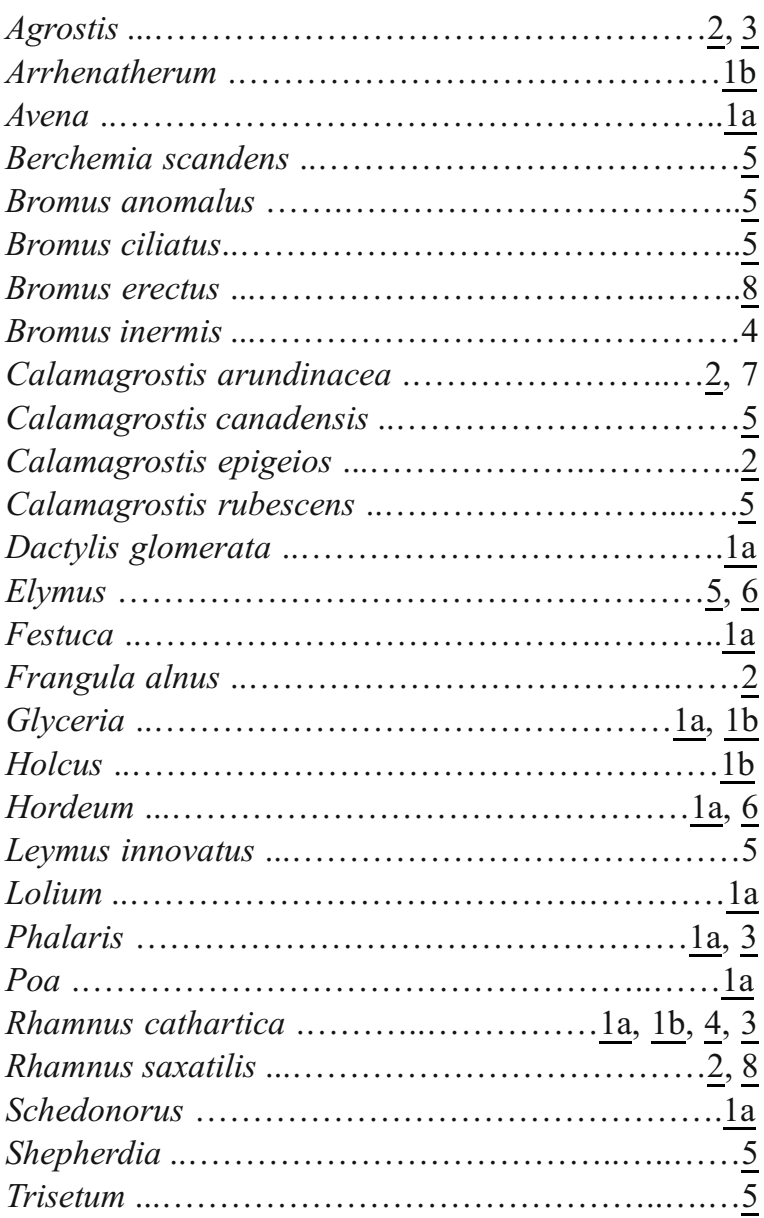


2. Telium

\subsection{Position}

2.1.1 Predominantly abaxial, almost no infection on adaxial .....................................5, 7

2.1.2 Infection on abaxial surface heavier ........2, 3

2.1.3 Amphigenuos ........................1, 4, 6, 8

\subsection{Loculation}

2.2.1 Not loculate $\underline{1 \mathrm{~b}}, \underline{4}, \underline{6}, 7,8$

2.2.2 Slightly loculate $\underline{1 \mathrm{~b}}, \underline{3}, \underline{4}, \underline{6,8}$

2.2.3 Clearly loculate $1 \mathrm{a}, \underline{3}, 5$

\subsection{Presence of paraphyses}

2.3.1 Abundant .5

2.3.2 Present, a few $1 \mathrm{a}, 1 \mathrm{~b}, 2$

2.3.3 Rarely observed $3,4,6,7$

2.3.4 Not observed . .8

\subsection{Shape of papraphyses}

2.4.1 Fused sheet-like 1a, $2, \underline{3}, 5$

2.4.2 Clavate or cylindrical $1 \mathrm{a}, 1 \mathrm{~b}, \underline{3}, 4,6,7$

3. Teliospore

\subsection{Shape of spores}

3.1.1 Clavate $\underline{1 \mathrm{a}}, \underline{1 \mathrm{~b},}, 2, \underline{3}, \underline{4}, \underline{6}, 7,8$

3.1.2 Short clavate $1 \mathrm{a}, 1 \mathrm{~b}, 3,4,5$

3.1.3 Obovoid $\underline{1 \mathrm{a}}, \underline{\mathrm{b}, 3,3,5}$

3.2 Presence of 3-celled spore

3.2.1 Common .................................. 8

3.2.2 Occasionally observed ........................ 2

3.2.3 Not observed .............. a , 1b, 3, 4, 5, 6, 7

3.3 Presence of mesospore

3.3.1 Present $1 \mathrm{a}, 2,3,4,5,6,8$

3.3.2 Not observed $1 \mathrm{~b}, 7$

3.4 Mean length excluding digitations

3.4.1 Shorter than $45 \mu \mathrm{m}$ 3,4 ,

3.4.2 Medium 45-55 $\mathrm{mm}$............1a, 1b, 5, 6, 7, 8

3.4.3 Long, over $55 \mu \mathrm{m}$ .2

3.5 Mean width

3.5.1 Narrow $13-15 \mu \mathrm{m}$ $.2,4,8$

3.5.2 Medium 15-16 $\mu \mathrm{m}$ $1 b, 3,5,6,7$

3.5.3 Wide, over $16 \mu \mathrm{m}$ $1 \mathrm{a}$

\subsection{Mean width of hilum}

3.6.1 Narrow 5-6 $\mu \mathrm{m}$ $3,4,5,8$

3.6.2 Medium 6-6.5 $\mu \mathrm{m}$ $.2,6$

3.6.3 Wide, over $6.5 \mu \mathrm{m}$ $1 \mathrm{a}, 1 \mathrm{~b}$
3.7 Apical digitation

3.7.1 Poorly digitated, digitation toe-like; or mean length of the longest digitation shorter than $5 \mu \mathrm{m}$

3.7.2 Mean length of the longest digitation $6-8 \mu \mathrm{m}$ $2,3,7$

3.7.3 Mean length of the longest digitation $8-12 \mu \mathrm{m}$ $1 \mathrm{a}, 1 \mathrm{~b}, 4,8$

3.7.4 Mean length of the longest digitation over $12 \mu \mathrm{m}$ 6

\subsection{Septum}

3.8.1 Spore constrict at septum

3.8.2 Constriction not obvious …ㅁa, $\underline{1 \mathrm{~b}}, \underline{2}, \underline{4}, \underline{5}, \underline{6}, \underline{8}$

4. Uredinium (taxa 2 and 8 were not available for this state) 4.1 Size

4.1.1 Regular size $0.2-1.0 \times 0.1-0.2 \mathrm{~mm}$ $1 \mathrm{a}, 1 \mathrm{~b}, 4,5,6,8$

4.1.2 Look smaller than regular size, $0.1-0.5 \times$ $0.05-0.1 \mathrm{~mm}$ 3

\subsection{Position}

4.2.1 Predominantly adaxial $1 b, 3,8$

4.2.2 Amphigenous $1 \mathrm{a}, 4,5,6$

5. Urediniospore (taxa 2 and 8 were not available for this state)

5.1 Mean length

5.1.1 Shorter than $20 \mu \mathrm{m}$ $\ldots 4$

5.1.2 Medium 20-22 $\mu \mathrm{m}$ $1 b, 3,6,8$

5.1.3 Longer than $22 \mu \mathrm{m}$ 1a, 5

\subsection{Mean width}

5.2.1 Narrower than $18 \mu \mathrm{m}$ $1 b, 3,4$

5.2.2 Medium 18-19 $\mu \mathrm{m}$ $1 \mathrm{a}, 6,8$

5.2.3 Wider than $19 \mu \mathrm{m}$ .5

1. P. coronata var. avenae Fraser \& Ledingham, Scientific Agriculture (1933) 13:313-323. (Figs. 4a-z; Clades IV and V)

Uredinia amphigenous or predominantly on adaxial leaf surface, $0.2-0.8 \times 0.1-0.2 \mathrm{~mm}$, covered or ruptured (Fig. 4a), paraphyses cylindrical; spores $15-29$ (32) $\times(11)$ 15-25 $\mu \mathrm{m}$, germ pores 5-15, scattered, vague, some show invagination or cuticular caps in congo-red stain. Telia often covered on abaxial leaf surface, ruptured on adaxial, 0.1$0.4 \times 0.1-0.2 \mathrm{~mm}$, ovoid to spherical, merged into long strips or irregular shapes, loculated or slightly loculated; teliospores variable, obvoid, short-clavate and clavate, not 
constricted or constricted at septa, some with a bump close to septum in lower cell, (28) 30-75×(9) 11-21 (24) $\mu \mathrm{m}$; digitations of various shapes, either long and sinuous or branched, medium length and sturdy, short and bump-like, or otherwise irregularly formed; the longest digitations per spore (3) 6-16 (20) $\mu \mathrm{m}$; pedicel remnants hyaline to brownish, hila (3) $5-10 \mu \mathrm{m}$.

Lectotype: on Avena sativa, leg. Fraser, Canada, Saskatchewan, Saskatoon, July 25, 1923 (PUR 22125). Lectotype designated by Cummins (1971). Type seen!

1a. P. coronata var. avenae f. sp. avenae Urban \& Marková, Acta Universitatis Carolinae (1993) 37:93147 (Figs. 4a-o; Clade V)

Uredinia amphigenous, spores $15-29(32) \times 13-25 \mu \mathrm{m}$, most cases with $9-15$ germ pores, usually show clear invagination or culticular caps in congo-red stain (Fig. 4j), occasionally $5-8$ obscure pores. Telia often clearly loculated (Fig. 4d, i); teliospores (28) 30-67 (72) × 12-21 (24) $\mu \mathrm{m}, 1$-celled spores observed.

Host: on Avena, Dactylis, Hordeum, Festuca, Glyceria, Lolium, Phalaris, Poa and Schedonorus.

Distribution: cosmopolitan.

Other specimens examined: Afghanistan, intercepted in New York?, on Lolium perenne, 1976 (BPI 060333) Austria, Bergenland, Gussing, 'S der Strabe nach Sulz am N-Ufer des Fischteiches', on Glyceria maxima, 31 Aug. 1981, J. Haifellner \& J. Poelt (B 70 0012413). Styria, south-east of Stubenberg, 'beim SchloB Schielleiten, Ufer des groBen Badeteiches (Gelande der Bundessportschule), on Glyceria sp., 23 Aug. 1983, J. Poelt \& H. Pittoni (B 70 0012414) - Canada, Ontario, Niagara fall, Vineland station, bank of the lake Ontario, 20 Aug. 2006, M. Liu (DAOM 240723) - Columbia, Intercepted in Miami Florida \#10464, on Hordeum sp., 15 Jan 1960 (BPI 060255) Greece, Isle of thasos, Skala Potamia, on Avena sp., 21 May 2000, A. Andrews (K(M): 77013) - Hungary, 'comit. Vas, ad/haud procul opp. Köszeg', on Glyceria maxima, 21 Oct. 1986 S. Tóth (BP 91486). 'Comit. Pest, pr. Pag. Cinkota ad lacum "Naplás-tó" in Phragmitetum', on Glyceria maxima, 10 Oct 1993, E. I. Simay (BP 88886). 'Comit. Vas, in agris pr. Pag. Torony haud procul opp. Szombathely', on Lolium multiflorum, 21 Oct. 1982, S. Tóth (BP 87214). 'Comit. Pest, in pratis udis ad rivulum Rákospatak, immediate infra opp. Gödöllö', on Glyceria maxima, 24 Sept. 1980, S. Tóth (BP 87217). 'Comit. Veszprém, pr. Rivulum Bakony-ér: haud procul pagi Pápakovácsi', on Avena sativa, 3 July 1986 S. Tóth \& A. Horánszky (BP 91484). 'Comit. Pest, pr. Pag. Tápiószele in agris.', on Avena sativa, 5 Oct. 1988, E. I. Simay (BP 88887) - Sweden, Gastrikland, valbo, on Avena sativa, 7 Sept. 2003, G. Odelvik \& B. Hellstrom ((S)reg.nr. F 35766) — United States, Minnesota, Rock
Creek State Park, west of Grinnell, Jasper Co., on Schedonorus phoenix, 21 Sept. 1995, A. P. Roelfs \& J. W. McCain (PUR N1015). North Dakota, Cass Co., Fargo, on Avena sativa, 29 Sept. 2004, R. W. Stack (BPI 871776). Virginia, Montgomery Co., Blacksburg 607 Lucas Dr., alt. $2175 \mathrm{ft}$, on Schedonorus phoenix, 04 Oct. 2004, C. W. Roane (BPI 871669).

1b. P. coronata var. avenae f. sp. graminicola Urban \& Marková, Acta Universitatis Carolinae (1993) 37:93147 (Figs. 4p-z; Clade IV)

Uredinia predominantly adaxial, spores $16-28(31) \times(11)$ $15-22 \mu \mathrm{m}$, germ pore $6-11$, invagination not clear and no culticular caps in congo-red (Fig. $4 \mathrm{w}, \mathrm{x}$ ). Telia slightly to no loculated, teliospores (30) 34-75×(9) 11-19 (21) $\mu \mathrm{mm}, 1$ celled spores not observed.

Host: Arrhenatherum elatius, Glyceria and Holcus.

Distribution: North America, South America, Europe.

Specimens examined: England, Middlesex, Ealing, Perivale Wood, on Glyceria maxima, 05 Oct. 2002, A. Henrici (K(M): 104797). Berkshire, Windsor Great Park; Bishopsgate entrance, on Holcus lanatus, 18 Aug. 2000, N. W. Legon (K(M): 70209). South Somerset, Staple Common (Blackdown Hills); near Castle Neroche, on Holcus sp., 15 July 2003, N. W. Legon (K(M): 115658) - Hungary, "montes Bükk-hegység, ad margines silvarum "Bolhás" pr. Fontem Jávor-kut', on Arrhenatherum elatius, 09 Sept. 1982, S. Tóth (BP 91761) - United States, Hawaii, Big island, Mauna Loa Road above Hawaii Volcanoes National Park, alt. $1000 \mathrm{~m}, 19^{\circ} 26^{\prime} 281^{\prime \prime} \mathrm{N}, 155^{\circ} 18^{\prime} 334^{\prime \prime} \mathrm{W}$, on Holcus lanatus, 05 Aug. 2005, M. Schöller MCA2958 (BPI 871068). California, Redwood National Park, Bald Hill Prairies, on Arrhenatherum elatius, 01 Oct. 1991 (PUR N5407). Virginia, Montgomery, Blacksburg, on Arrhenatherum elatius, 27 Jun. 1990, C. W. Roane (PUR N5409).

Discussion: Puccinia coronata var. avenae was first designated by Fraser and Ledingham (1933), and accepted by Cummins (1971) and Urban and Marková (1993). We examined and sequenced the type specimen (PUR 22125), which grouped in Clade V of our phylogenetic hypotheses. Urban and Marková (1993) further separated P. coronata var. avenae into two formae speciales, noting that $\mathrm{f}$. sp. avenae was narrowly specialized on cultivated and other oat species, while f. sp. graminicola was specialized on $A r$. elatius and $R$. carthatica. Both our ITS tree (with extensive sampling) and combined tree provide support for this partition (samples on Avena grouped in Clade $\mathrm{V}$ and those on Ar. elatius in Clade IV). Although Clades IV and V were strongly supported as sister clades in our combined gene tree (Fig. 2), Clade IV received strong individual support but not Clade $\mathrm{V}$. Therefore, the combination of these two groups is recognized as $P$. coronata var. avenae. However, the statement by Urban and Marková (1993) that oat crown rust was 
Table 4 Unbalanced two tail $T$-test of selected quantitative characters on pair-wise comparison of clades

\begin{tabular}{|c|c|c|c|c|c|c|c|c|c|c|}
\hline clade & Mean $(\mu \mathrm{m})$ & $\mathrm{n}$ & clade II & clade III & clade IV & clade V & clade VI & clade VII & clade VIII & clade IX \\
\hline \multicolumn{11}{|c|}{ Teliospore length } \\
\hline clade I & 45.6 & 128.0 & $<0.01$ & $<0.01$ & $<0.01$ & $<0.01$ & 0.153 & $<0.01$ & 0.229 & $<0.01$ \\
\hline clade II & 51.9 & 89.0 & & $<0.01$ & 0.161 & $<0.01$ & $<0.01$ & $<0.01$ & $<0.01$ & $<0.01$ \\
\hline clade III & 42.2 & 81.0 & & & $<0.01$ & $<0.01$ & $<0.01$ & $<0.01$ & $<0.01$ & $<0.05$ \\
\hline clade IV & 50.5 & 145.0 & & & & 0.180 & $<0.01$ & $<0.01$ & $<0.01$ & $<0.01$ \\
\hline clade V & 49.0 & 177.0 & & & & & 0.076 & $<0.01$ & $<0.05$ & $<0.01$ \\
\hline clade VI & 47.0 & 133.0 & & & & & & $<0.01$ & 0.825 & $<0.01$ \\
\hline clade VII & 59.0 & 107.0 & & & & & & & $<0.01$ & $<0.01$ \\
\hline clade VIII & 46.7 & 46.0 & & & & & & & & $<0.01$ \\
\hline clade IX & 40.0 & 162.0 & & & & & & & & \\
\hline \multicolumn{11}{|c|}{ Teliospore width } \\
\hline clade I & 15.4 & 144 & $<0.01$ & $<0.01$ & 0.948 & $<0.01$ & 0.416 & $<0.01$ & 0.142 & 0.788 \\
\hline clade II & 13.7 & 81 & & 0.294 & $<0.01$ & $<0.01$ & $<0.01$ & 0.079 & $<0.01$ & $<0.01$ \\
\hline clade III & 13.3 & 79 & & & $<0.01$ & $<0.01$ & $<0.01$ & $<0.01$ & $<0.01$ & $<0.01$ \\
\hline clade IV & 15.4 & 158 & & & & $<0.01$ & 0.461 & $<0.01$ & 0.137 & 0.849 \\
\hline clade V & 16.7 & 179 & & & & & $<0.01$ & $<0.01$ & $<0.01$ & $<0.01$ \\
\hline clade VI & 15.7 & 108 & & & & & & $<0.01$ & 0.044 & 0.538 \\
\hline clade VII & 14.1 & 106 & & & & & & & $<0.01$ & $<0.01$ \\
\hline clade VIII & 15.0 & 46 & & & & & & & & 0.079 \\
\hline clade IX & 15.5 & 158 & & & & & & & & \\
\hline \multicolumn{11}{|c|}{ Teliospore hilum width } \\
\hline clade I & 6.4 & 119 & $<0.01$ & $<0.01$ & $<0.01$ & $<0.01$ & $<0.01$ & $<0.05$ & $<0.01$ & $<0.01$ \\
\hline clade II & 5.4 & 79 & & 0.741 & $<0.01$ & $<0.01$ & 0.138 & $<0.01$ & $<0.05$ & 0.126 \\
\hline clade III & 5.4 & 58 & & & $<0.01$ & $<0.01$ & 0.398 & $<0.01$ & $<0.05$ & 0.372 \\
\hline clade IV & 7.0 & 185 & & & & 0.256 & $<0.01$ & $<0.01$ & $<0.01$ & $<0.01$ \\
\hline clade V & 6.8 & 227 & & & & & $<0.01$ & $<0.01$ & $<0.01$ & $<0.01$ \\
\hline clade VI & 5.5 & 193 & & & & & & $<0.01$ & $<0.01$ & 0.938 \\
\hline clade VII & 6.1 & 97 & & & & & & & $<0.01$ & $<0.01$ \\
\hline clade VIII & 5.0 & 31 & & & & & & & & $<0.01$ \\
\hline clade IX & 5.5 & 154 & & & & & & & & \\
\hline \multicolumn{11}{|c|}{ Teliospore longest digitation } \\
\hline clade I & 12.6 & 108 & $<0.01$ & $<0.01$ & $<0.01$ & $<0.01$ & $<0.01$ & $<0.01$ & $<0.01$ & $<0.01$ \\
\hline clade II & 8.5 & 79 & & $<0.01$ & 0.194 & 0.441 & $<0.01$ & $<0.01$ & $<0.01$ & $<0.01$ \\
\hline clade III & 10.9 & 83 & & & $<0.01$ & $<0.01$ & $<0.01$ & $<0.01$ & $<0.01$ & $<0.01$ \\
\hline clade IV & 8.9 & 172 & & & & 0.525 & $<0.01$ & $<0.01$ & $<0.01$ & $<0.01$ \\
\hline clade V & 8.7 & 180 & & & & & $<0.01$ & $<0.01$ & $<0.01$ & $<0.01$ \\
\hline clade VI & 4.7 & 154 & & & & & & $<0.01$ & $<0.01$ & $<0.01$ \\
\hline clade VII & 7.2 & 125 & & & & & & & 0.621 & 0.058 \\
\hline clade VIII & 7.0 & 111 & & & & & & & & 0.299 \\
\hline clade IX & 6.7 & 210 & & & & & & & & \\
\hline \multicolumn{11}{|c|}{ Urediniospore length $^{\mathrm{a}}$} \\
\hline clade & mean & $\mathrm{n}$ & clade II & clade III & clade IV & clade $\mathrm{V}$ & clade VI & clade IX & & \\
\hline clade I & 20.5 & 143.0 & $<0.01$ & $<0.01$ & $<0.05$ & $<0.01$ & $<0.01$ & $<0.05$ & & \\
\hline clade II & 21.4 & 45.0 & & $<0.01$ & 0.669 & $<0.05$ & $<0.01$ & 0.456 & & \\
\hline clade III & 19.4 & 67.0 & & & $<0.01$ & $<0.01$ & $<0.01$ & $<0.01$ & & \\
\hline clade IV & 21.2 & 102.0 & & & & $<0.01$ & $<0.01$ & 0.796 & & \\
\hline clade V & 22.2 & 197.0 & & & & & 0.051 & $<0.01$ & & \\
\hline clade VI & 22.8 & 76.0 & & & & & & $<0.01$ & & \\
\hline clade IX & 21.1 & 103.0 & & & & & & & & \\
\hline
\end{tabular}


Table 4 (continued)

\begin{tabular}{|c|c|c|c|c|c|c|c|c|c|}
\hline clade & Mean $(\mu \mathrm{m})$ & $\mathrm{n}$ & clade II & clade III & clade IV & clade $\mathrm{V}$ & clade VI & clade VII & clade IX \\
\hline \multicolumn{10}{|c|}{ Urediniospore width ${ }^{a}$} \\
\hline clade I & 18.3 & 143.0 & 0.063 & $<0.01$ & $<0.01$ & 0.239 & $<0.01$ & $<0.01$ & \\
\hline clade II & 18.8 & 45.0 & & $<0.01$ & $<0.01$ & 0.446 & $<0.01$ & $<0.01$ & \\
\hline clade III & 17.3 & 67.0 & & & 0.203 & $<0.01$ & $<0.01$ & 0.491 & \\
\hline clade IV & 17.7 & 102.0 & & & & $<0.01$ & $<0.01$ & 0.333 & \\
\hline clade V & 18.6 & 197.0 & & & & & $<0.01$ & $<0.01$ & \\
\hline clade VI & 19.7 & 76.0 & & & & & & $<0.01$ & \\
\hline clade IX & 17.5 & 103.0 & & & & & & & \\
\hline
\end{tabular}

${ }^{\text {a }}$ Urediniospore specimens were not available for clade VII and VIII, thus only seven clades were compared

specialized only on oats was not supported. Based on our sampling and genes analysed, $P$. coronata var. avenae f. $\mathrm{sp}$ avenae has a wide host range, i.e. 7 genera in 3 tribes while $f$. sp graminicola occurs not only on Ar. elatius, but also on Holcus spp and Glyceria spp.

Both f. spp. are characterized by having relatively wide hila and variable teliospores. Telia of $P$. coronata var. avenae f. sp. avenae are more clearly loculated (Figs. 4d, i) than f. sp. graminicola (Figs. $4 \mathrm{u}, \mathrm{z}$ ). Urediniospore germ pore invaginations and cuticular caps are usually present in the former (Fig. 4j) but less often in the later (Figs. 4w, x).

2. P. coronata Corda Icones fungorum hucusque cognitorum (1837) 1:6 var coronata (Figs. 51, m; Clade VII)

Uredinia not observed. Telia amphigenous, but abaxial infection heavier, covered or with a longitude rupture, $0.2-$ $0.4 \times 0.1-0.2 \mathrm{~mm}$, ovoid, merge to irregular shapes, slightly loculated; paraphyses fused; teliospores clavate to long clavate (Fig. 5m), (27) $32-80(84) \times 11-19 \mu \mathrm{m}$, often with long lower cells, average around $32 \mu \mathrm{m}, 3$-celled spores present (Fig. 51), digitations tuberculate, cylindrical, bifurcate, the longest per spore 4-13 (15) $\mu \mathrm{m}$, pedicel hilum 4-8 $\mu \mathrm{m}$.

Lectotypus (hic designatus): Icones fungorum hucusque cognitorum (1837) 1:6 Tab II fig 96.

Epitypus (hic designatus): PRM 155608, reported to be 'in foliis Luzulae albidae prope Reichenberg' by Corda (1837). The host was redetermined to be Calamagrostis arundinacea or Cal. villosa by M. Deyl (II 1961, note with specimen), while previously it was suggested to be Bromus erectus (Mühlethaler 1911) which was contested by I. Jørstad (May 1949, note with specimen). 'Reichenberg' is probably Liberec, Bohemia according to the current label, leg. \& det. Corda. Type seen!

Host: Agrostis stolonifera, Calamagrostis arundinacea seu villosa, and Calamagrostis epigeios.

Table 5 Grouping of the clades based on statistical tests on selected quantitative morphological characters

\begin{tabular}{|c|c|c|c|c|c|}
\hline teliospore length $(\mu \mathrm{m})^{\mathrm{a}}$ & $\begin{array}{l}\mathrm{VII}> \\
59\end{array}$ & $\begin{array}{l}\text { I, II, IV, V, VI,VIII }> \\
45<\mathrm{M}<52\end{array}$ & $\begin{array}{l}\text { III > } \\
42\end{array}$ & $\begin{array}{l}\text { IX } \\
40\end{array}$ & \\
\hline teliospore width $(\mu \mathrm{m})$ & $\begin{array}{l}\mathrm{V}> \\
16.7\end{array}$ & $\begin{array}{l}\text { I, IV, VI, VIII, IX }> \\
15<\mathrm{M}<16\end{array}$ & $\begin{array}{l}\text { II, III, VII } \\
13<\mathrm{M}<15\end{array}$ & & \\
\hline teliospore hilum width $(\mu \mathrm{m})$ & $\begin{array}{l}\mathrm{IV}, \mathrm{V}> \\
6.5<\mathrm{M}\end{array}$ & $\begin{array}{l}\mathrm{I}> \\
6.36\end{array}$ & $\begin{array}{l}\text { VII }> \\
6.1\end{array}$ & $\begin{array}{l}\text { II, III, VI, IX }> \\
5<\mathrm{M}<6\end{array}$ & $\begin{array}{l}\text { VIII } \\
5.0\end{array}$ \\
\hline teliospore longest digitations $(\mu \mathrm{m})$ & $\begin{array}{l}\text { I }> \\
12.6\end{array}$ & $\begin{array}{l}\text { III }> \\
10<\mathrm{M}<12\end{array}$ & $\begin{array}{l}\text { II, IV, V > } \\
8<\mathrm{M}<10\end{array}$ & $\begin{array}{l}\text { VII, VIII, IX }> \\
6<\mathrm{M}<8\end{array}$ & $\begin{array}{l}\mathrm{VI} \\
\mathrm{M}<5\end{array}$ \\
\hline urediniospore length $(\mu \mathrm{m})$ & $\begin{array}{l}\mathrm{V}, \mathrm{VI}> \\
22<\mathrm{M}\end{array}$ & $\begin{array}{l}\text { II, IV, IX > } \\
21<\mathrm{M}<22\end{array}$ & $\begin{array}{l}\mathrm{I}> \\
20<\mathrm{M}<21\end{array}$ & $\begin{array}{l}\text { III } \\
\mathrm{M}<20\end{array}$ & \\
\hline urediniospore width $(\mu \mathrm{m})$ & $\begin{array}{l}\mathrm{VI}> \\
19.7\end{array}$ & $\begin{array}{l}\text { I, II, V }> \\
18<\mathrm{M}<19\end{array}$ & $\begin{array}{l}\text { III, IV, IX } \\
\mathrm{M}<18\end{array}$ & & \\
\hline
\end{tabular}

${ }^{a}$ Roman letters stand for the clades; M: mean, >: significantly larger than the next category. 


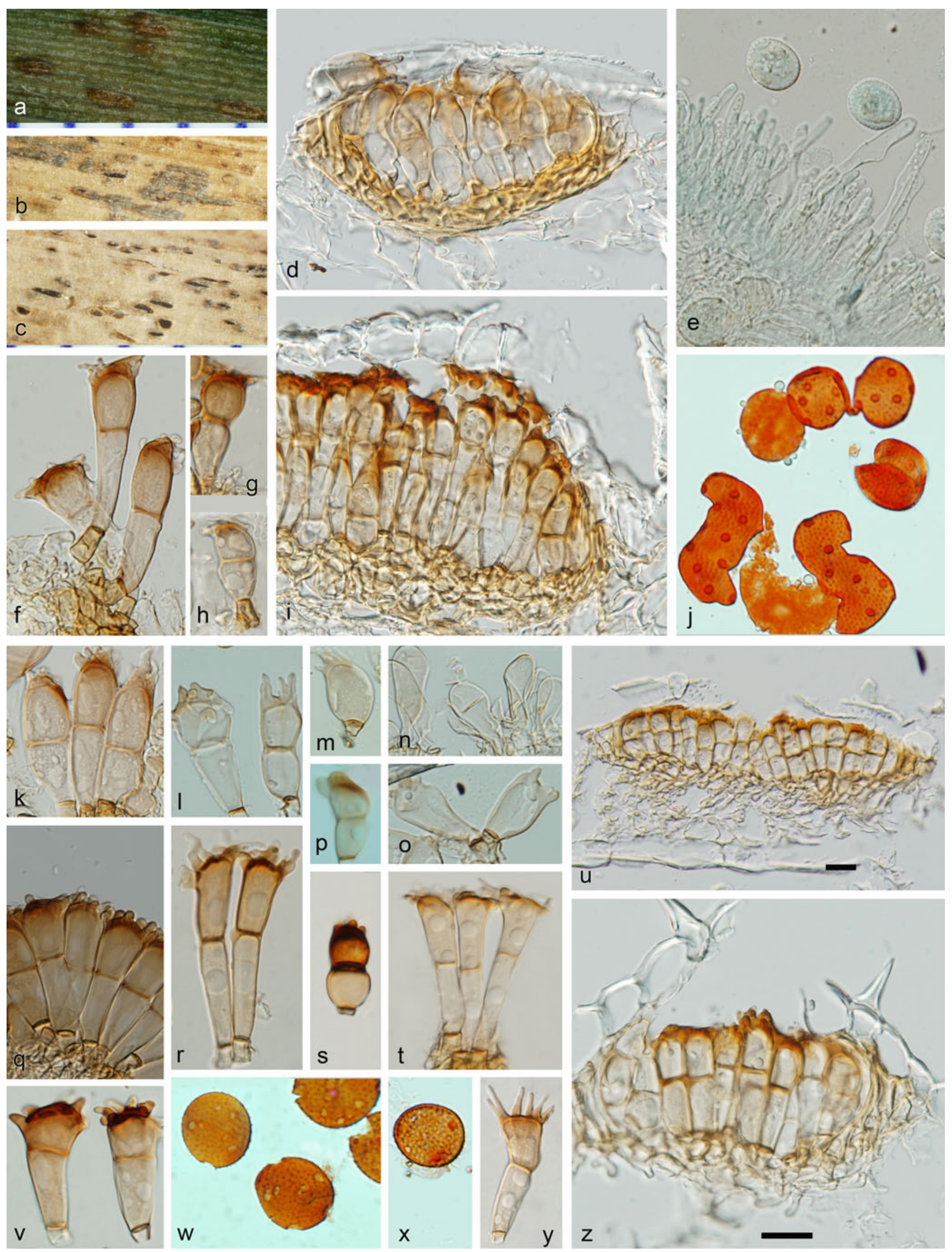


Fig. 4 Telial and uredinial states of $\boldsymbol{P}$. $\boldsymbol{c}$. var. avenae. a-o: $P$. $c$. var. avenae f. sp. avenae; $\mathbf{p}-\mathbf{z}: \boldsymbol{P}$. c. var. avenae f. sp. graminicola. a. uredinia with longtitudinal rupture (BPI 871766). b. telia of abaxial leaf surface mostly covered (BP 91486); c. telia on adaxial leaf surface often ruptured (BP 91486); $\mathbf{d}$ and i. loculated telia (PUR N1015); $\mathbf{f}-\mathbf{h}$, $\mathbf{k}-\mathbf{0}$ : teliospores of various shapes (f: B 70 0012413, G, h: BPI 871669); e. uredinospores and paraphyses (BPI 871766); j. urediniospores showing cuticular caps and invaginations in congo-red stain $(\mathrm{K}$ (M):77013) $\mathbf{p}-\mathbf{t}, \mathbf{v}, \mathbf{y}$ showing variable shapes of teliospores (BP 91761, PUR N5409, K(M):104797, BP 91761, K(M):104797); u, z showing telia slightly loculated (K(M):115658, K(M):115658); w urediniospore germ pores with no invagination and cuticular caps in congo-red stain $(\mathrm{K}(\mathrm{M}): 115658)$; $\mathbf{x}$ urediniospore germ pores with slight invagination and cuticular caps in congo-red stain ( $K$ (M):70209). For $\mathbf{a}, \mathbf{b}$ and $\mathbf{c}, 1$ unit $=1 \mathrm{~mm}, \mathbf{d}-\mathbf{z}$ (except $\mathbf{u}$ ) share the same scale, scale bar $=20 \mu \mathrm{m}$

Alternate host: Frangula alnus, Rhamnus saxatilis.

Distribution: Austria, Belgium, Bohemia, England, Germany, Hungary, Sweden.

Other specimens examined: Belgium, on Agrostis stolonifera, 13 Aug. 1988, De Meulder 1661 (BR 3933-53) Hungary, 'montes Mátra-hegység, in mte Sombokor hegy pr. Tabernam recreandi Honvéd-üdülő', on Calamagrostis epigeios,

20 Sept 1989, S. Tóth (BP 89848). 'montes Mátrahegység, in mte Hegyes-hegy ad locum otii Fallóskut haud [procul pagi Mátrakeresztes', 05 Sept 1991, S. Tóth (BP 89076).

Discussion: The epitype designated here has been considered to be the holotype by Urban (1966 note with specimen; 1967) and by Kaufman (1967). However the specimen lacks collection date data. Corda's (1837) illustration is the only reliable original material and it is hereby designated as lectotype with PRM 155608 designated as its epitype. The type variety is distinguished by having 3-celled teliospores, only observed otherwise in P. coronatilongispora. The name was previously considered as a catch-all for crown rust fungi excluded from other named varieties, and consequently credited with a wide host range, i.e. 48 grass genera (Cummins 1971; Urban and Marková 1993). Our molecular data do not support this scenario: the type specimen grouped in a well-supported and cohesive Clade VII. It may be endemic in Europe, with a telial host range mainly restricted to Cal. epigeios and Cal. arundinacea. One of four telial collections sampled here was collected on Agrostis from Belgium. DNA verification of the host identification was attempted but was not successful. The more restricted host range reported here may be partly due to sampling bias since many grass genera listed by Cummins (1971) and Urban and Marková (1993) were not sampled in our data, such as Aegilops, Alopecurus, Ammophila, Anthoxanthum, Apera, Beckmannia, Catabrosa, Cinna, Corynephorus, Cynodon, Cynosurus, Deschampsia, Hierochloë, Koeleria, Lamarkia, Melica, Milium, Molinia,
Parapholis, Phleum, Phragmites, Polypogon, Scolochloa, Sesleria, Setaria and Vulpia. On the other hand, specimens on numerous hosts previously documented for this variety were excluded, grouping in other clades. For instance, Agropyron repens (current name: Elymus repens; GRIN), Elymus and Hordeum (Clade I), Bromus (Clade II, III and VI), Arrhenatherum and Holcus (Clade IV), Festuca, Lolium, Poa (Clade V), Glyceria (Clade IV and V), Agrostis spp. (Clade IX), Cal. canadensis (Clade VI). For some specimens on Brachypodium, Helictrichon, Phalaris, Trisetum, relationships to the varieties described here are uncertain. Additional sampling of hosts not represented here will help to clarify the host range and distribution of the type variety of the species.

3. P. coronati-agrostidis Liu \& Hambleton sp. nov. (Figs. 5b, c, s; Clade IX; MycoBank \#: MB563543)

Puccnia coronata affinis sed teliosporis brevisibus et obesis, (22) 27-56 (61) $\times 11-20(22) \mu \mathrm{m}$, coronatis digitiformis tenuis, septis constrictorum differt.

Holotypus: on Agrostis stolonifera, Finland, 'Regio aboensis $(\mathrm{Ab} / \mathrm{V})$. Västanfjärd: Södersundvik, Grundsund. Humous-sandy littoral meadow, abundant', 05 Aug. 1977, H. Roivainen (PUR N114).

Etymology: Refers to the predominant telial host association for this variety.

Uredinia amphigenous but often adaxial, $0.1-0.5 \times$ $0.1 \mathrm{~mm}$, small, fusiform, ruptured; spores $16-26(28) \times(12)$ $15-21 \mu \mathrm{m}$; germ pores (4) 5-10 (11), invagination and caps vague or clear in congo-red stain. Telia mostly abaxial, or on stem, $0.1-0.4 \times 0.1-0.2 \mathrm{~mm}$, ovoid to cylindrical, slightly rise or submerged, mostly covered, slight to medium loculated (Fig. 5s); telio-paraphyses rare, clavate, barely fused; teliospores obvoid to clavate, constrict at septa (Figs. 5b, c), (22) $27-56(61) \times 11-20(22) \mu \mathrm{m}$; digitations tuberculate to cylindrical, longest per spore (2) 3-13 $\mu \mathrm{m}$; hila (3) 4-7 (8) $\mu \mathrm{m}$.

Host: Agrostis gigantea, Agrostis stolonifera, Phalaris arundinacea.

Distribution: Austria, Belgium, Canada, England, Finland, United States.

Other specimens examined: Austria, Viennese Basin (Wiener Becken), Gramatneusiedl (at or near the train station), $8^{\circ} 02^{\prime} \mathrm{N}, 16^{\circ} 29^{\prime} \mathrm{E}$, on Agrostis stolonifera, 23 Aug. 1994, Th. Barta (K(M): 60529) - Canada, British Columbia, [Victoria], Royal Oak Drive, roadside near Lochside Drive, on Agrostis gigantean, 09 Oct. 1960, G. B. Cummins (PUR 59741). Vancouver, University of British Columbia, near tennis court, on Phalaris arundinacea, 19 Aug. 1994, A. P. Roelfs (PUR 1268) — England, Berkshire, Windsor Great Park; Bishops gate entrance, on Agrostis tenuis?, 18 Aug. 2000, N. W. Legon (K(M): 82517) - United States, Wisconsin, Dane, Madison, on Agrostis gigantean, 30 July 


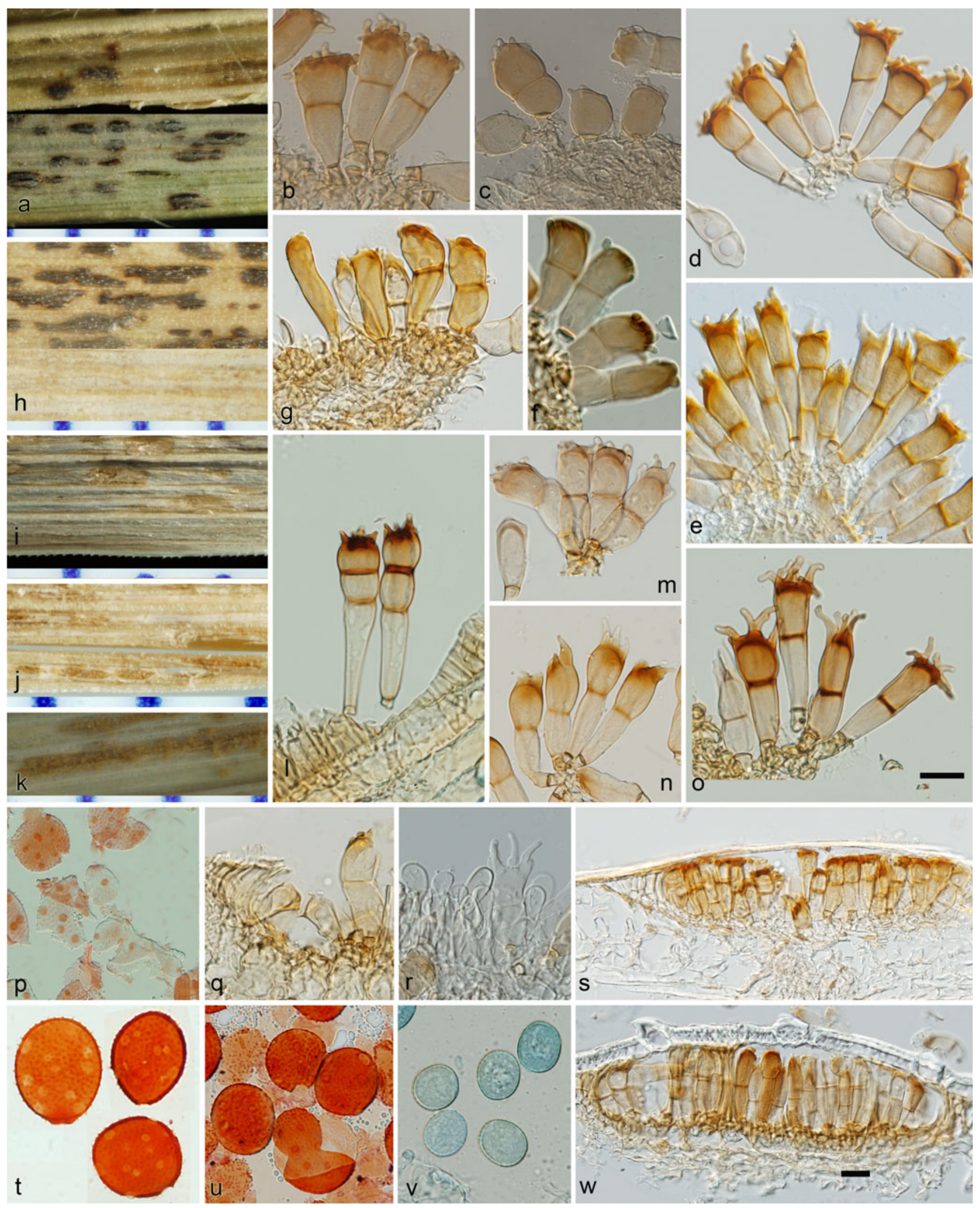


Fig. 5 Morphological features of species other than $P$. $c$. var. avenae. P. c. var coronata: variation of teliospores; l: 3-celled spores (BP 89848); m: 1-celled and normal spores (PRM155608 type). $\boldsymbol{P}$. coronati-agrostis: b, c: ovoid to clavate teliospores (PUR 55024, PUR N114 type); s: slightly loculated telia (K(M):82517). $\boldsymbol{P}$. coronati-brevispora: $\mathbf{d}$ : short spores (compare with e) (DAOM 240183); i: uredinia covered (PUR N1371); p: clear cuticular caps (DAOM 240183). P. coronati-calamagrostidis: f, g: poor digitations (PUR 57282, BPI 747648); h: telia predominantly on abaxial (lower) not adaxial (upper) surface of the leaf (BPI 1100292); j: open uredinia (BPI 59821); q: fused telia paraphyses (PUR N2268); t: invagination not clear in congo-red stain (BPI 59821); w: well loculated telia (PUR N2268). P. coronati-hordei: $\mathbf{k}$ : uredinia with spores piled on top (PUR 89857 type); o: teliospores with long digitations (PUR 89857 type); $\mathbf{r}$ : cylindrical paraphyses (PUR N1396); u: germ pores with clear invagination and cuticular caps visible in congo-red stain but not in cottonblue (v) (PUR 89875 type). P. coronati-japonica: $\mathbf{n}$ : teliospores contricted at septa (PUR F16131 type). P. coronati-longispora: a: telia covered on abaxial (lower) and ruptured on adaxial (upper) leaf surface (PRC 194); e: longer teliospores (compare with d) (PRC 196 type). For $\mathbf{a}, \mathbf{h}, \mathbf{i}, \mathbf{j}$, and $\mathbf{k}, 1$ unit $=1 \mathrm{~mm}, \mathbf{b}-\mathbf{g} ; \mathbf{l}-\mathbf{r}, \mathbf{t}-\mathbf{v}$ share the same scale, $\mathbf{s}$ and w share same scale, scale bar $=20 \mu \mathrm{m}$

1955, H. C. Greene (PUR 54591). Wyoming, Albany, Wheatland Cutoff, west end of Wildlife Station, Laramie Mountains, alt. 6200 ft., on Agrostis gigantea, 01 Oct. 1955, W. G. Solheim \& Mycology class (PUR 55024).

Discussion: Teliospores are short and plump, digitations short and thin. All but one telial specimen ( 9 of 10) examined were collected on Agrostis spp. suggesting a possible misdetermination of the host for the only specimen on Phalaris. Although Phalaris is easily distinguished from Agrostis, the specimen does not include diagnostic plant parts. In our experience sequencing of the host DNA has proved successful for confirming herbarium label identifications but in this case our attempts to sequence the matK gene were unsuccessful.

4. P. coronati-brevispora Liu \& Hambleton sp. nov. (Figs. 5d, i, p; Clade III; MycoBank \#: MB563541)

Teleosporis parvis, (29) 32-53 (56) x 10-17 (19) $\mu \mathrm{m}$, in Bromus cognit.

Holotypus: on Bromus inermis, United States, Wisconsin, Columbia, breeding nurseries at the Univ. of Wisconsin Arlington Agricultural Research Station near Arlington, 01 Sept. 1999, C. R. Grau WPc-95 (PUR N652).

Etymology: Refers to the shorter size of teliospores compared with $P$. coronati-longispora.

Uredinia amphigenous, often produce light infection on both sides of the leaf, $0.3-1.0 \times 0.1-0.2 \mathrm{~mm}$, often covered (Fig. 5i), some ruptured longtitude; spores $14-24 \times(11) 13-$ $22 \mu \mathrm{m}$, germ pores (7) 8-12 (14), scattered, cuticular caps clear in congo-red stain (Fig. $5 \mathrm{p}$ ). Telia amphigenous, light infection on both sides, $0.1-0.4(0.7) \times 0.1-0.2 \mathrm{~mm}$, not to slightly loculated, telio-paraphyses rarely observed, clavate, to cylindrical; teliospores, short clavate to clavate (Fig. 5d) (29) $32-53(56) \times 10-17$ (19) $\mu \mathrm{m}$, digitations cylindric wriggling, branched, slightly tapering, longest per spore (5) 7-17 (20) $\mu \mathrm{m}$, pedicel hila 4.0-7.5 (9.0) $\mu \mathrm{m}$.

Host: Bromus inermis.

Distribution: Canada, United States.

Other specimens examined: Canada, Ontario, Hastings, west of Trenton, on west side of Wooler Rd just north of Old Hwy 2, growing in oat field in production, on Bro. inermis, 12 July 2006 S. Hambleton, M. Liu, T. Fetch (DAOM 240063). Niagara Fall, Vineland Station, bank of the Lake Ontario, 20 Aug. 2006, M. Liu (DAOM 240183) - United States, Wisconsin, greenhouse generation of culture collected August 1996 at Univ. Wisconsin research station, Arlingtion, on Bro. inermis, 01 Mar. 1997, N. Delgado (PUR N1371).

Discussion: See below under $P$. coronati-longispora.

5. P. coronati-calamagrostidis Liu \& Hambleton nom. et stat. nov. (Figs. 5f-h, j, q, t, w; Clade VI; MycoBank \#: MB563544)

$\equiv$ Puccinia coronata Corda var. calamagrostis Fraser \& Ledingham, Sci. Agric. 13:315 (1933), non Puccinia calamagrostidis P. Sydow Ured. Exsic. 13-15: no. 662 (1892)

Lectotypus (hic designatus): on Calamagrostis canadensis, Canada, Manitoba, Dauphin, Sept. 1917, W. P. Fraser (PUR 22155).

Uredinia often abaxial, opened with spores piled on top (Fig. 5j), fusiform or ellipsoid, $0.3-0.5 \times 0.1-0.2 \mathrm{~mm}$; spores $16-27 \times 15-25 \mu \mathrm{m}$, germ pores $4-10$, scattered, most cases invagination not clear and no caps shown in congo-red stain (Fig. 5t). Telia predominantly abaxial, almost no infection on adaxial surface (Fig. $5 \mathrm{~h}$ ), all covered, individual sorus look small, $0.1-0.3 \times 0.1 \mathrm{~mm}$, some merged into irregular stripes; loculated (Fig. $5 \mathrm{w}$ ), telio-paraphyses fused (Fig. 5q), sheet-like, orange; teliospores obvoid to short clavate $31-72(83) \times 10-21 \mu \mathrm{m}$, usually poorly digitated, digitations toe-like, some narrow cylindrical (Figs. 5f, g), longest per spore (1) $2-10$ (12) $\mu \mathrm{m}$, hila (2.5) $4.0-$ $8.0 \mu \mathrm{m}$.

Host: Bromus anomalus, Bromus ciliatus, Calamagrostis canadensis, Calamagrostis rubescens, Leymus innovatus, Trisetum canecens

Distribution: Canada, United States

Other specimens examined: Canada, Alberta, Bow River, Banff National Park Valley near Mt. Eisenhower, on Leymus innovatus, 21 Aug. 1953, J. A. Calder and D. B. O. Savile (BPI 1100292). Manitoba, Brandon, on Calamagrostis canadensis, 25 Aug. 1917, W. P. Fraser (PUR 22167). Manitoba, on Bromus ciliatus, 01 Sept. 1918, V. W. Jackson (DAOM 204923). Saskatchewan, Foam Lake, on Bromus ciliatus, 13 July 1922, J. W. Scannell (PUR 22132). Saskatoon, on Bromus ciliatus, 06 Aug. 1922, W. P. Fraser (PUR 22133) - United States, Montana, Specimen Creek, Gallatin Canyon, Big Sky area, on 
Calamagrostis rubescens, 11 Aug. 1982, G. B. Cummins (PUR 66551). Wisconsin, Sauk Co., near Leland, on Calamagrostis canadensis, 29 Aug. 1964, H. C. Greene (DAOM 107653). Burnett Co. Ca. 3 miles NW of Grantsburg. Ferry Road, edge of meadow, on Calamagrostis canadensis, 9 Sept. 1995 A. P. Roelfs (BPI 747648). Crex Meadows Wildlife Area N of Grantsburg, on Calamagrostis canadensis, 12 Aug. 2000, A. P. Roelfs, T. Crosby, J. W. McCain (PUR N2268). Wyoming, Gros Ventre Slide, near Jackson, on Bromus anomalus, 30 Aug. 1960, G. B. Cummins (PUR 57282).

Discussion: Both morphological and molecular evidence indicates that $\mathrm{P}$. coronata collections on Br. ciliatus and $\mathrm{Cal}$. canadensis are genetically alike and different from other taxa, thus we recognized the taxon at the species level and designated a lectotype from original material collected by Fraser in 1917. Host range is not restricted to these two species, but extends to other Bromus spp. and other grass genera. The species is distinctive for several characters: telia often predominantly present on the abaxial leaf surface; sori small and well-loculated, fused telial paraphyses, and teliospore digitations short and toe-like.

6. P. coronati-hordei Liu \& Hambleton nom. et stat. nov. (Figs. 5k, o, r, u, v; Clade I; MycoBank \#: MB563545)

$\equiv$ Puccinia coronata Corda var. hordei Jin \& Steffenson, Mycologia (1999) 91:878; non Puccinia hordei G. H. Otth Mitt.Naturf. Ges. Bern (1871)1870:114

Holotypus (hic designatus): on inoculated Hordeum vulgare, leg. Y. Jin JIN91-36, United States, North Dakota, Fargo, 46 $52^{\prime} 30^{\prime \prime} \mathrm{N} / 96^{\circ} 47^{\prime} 30^{\prime \prime} \mathrm{W}$, Mar. 29, 1992 (PUR 89857).

Uredinia amphigeous, $0.2-0.7 \times 0.1 \mathrm{~mm}$, covered, ruptured longtitude or opened, fusiform with urediniospore piled on top (Fig. 5k); spores 16-25 (27) $\times 15-22$ (24) $\mu \mathrm{m}$; germ pores (4) 6-11 (14), scattered or bizonate, wall invagination or culticular caps clear in congo-red stain (Fig. $5 \mathrm{u}$ ). Telia amphigenous, some with heavier infection on adaxial side, $0.2-0.4 \times 0.1 \mathrm{~mm}$, ovoid to cylindrical, mostly covered, not loculated to slightly loculated, telioparaphyses rarely observed (Fig. 5r); teliospores obvoid to clavate, 35-55 (57) $\times(10) 12-19(21) \mu \mathrm{m}$, digitations long, various shapes (Fig. 5o): bump like, long tapering, irregular shape wriggling or bifurcate, longest per spore (6) 7-18 (21) $\mu \mathrm{m}$, pedicel hilum 4.5-8 (10) $\mu \mathrm{m}$.

Host: Elymus and Hordeum.

Distribution: North America.

Other specimens examined: Canada, Ontario, Ottawa, Western Parkway W of Maitland Ave., on Elymus repens, 09 July 1982, J. A. Parmelee and D. B. O. Savile (DAOM 183691) - United States, Minnesota, Hennepin, roadside across from Flying Cloud Airport, Eden Prairie, on Elym. repens, 28 Sept. 1995, A. P. Roelfs and J. W. McCain (PUR N1426). North Dakota, Cass, barley field, Casselton, on
Elym. repens, 10 July 1992, A. P. Roelfs (PUR N1413). on Hordeum jubatum, 10 July 1992, A. P. Roelfs (PUR N1539). Wisconsin, Polk, St. Croix State Park, St. Croix Falls, on Elym. virginicus, 13 Sept. 1995, A. P. Roelfs (PUR N1358). Lion's Club Park, N edge of St. Croix Falls, on Elym. repens, 14 Sept. 1995, A. P. Roelfs (PUR N1406). Osceola, glen below Cascade Falls, on Elym. hystrix, 25 July 1996, J. W. McCain (PUR N1396).

Discussion: Barley crown rust was described by Jin and Steffenson (1999) and is distinguished by having long teliospore digitations and a restricted host association. The authors observed that the morphology of this fungus is very similar to the taxon Peturson (1954) described as P. coronata f. sp. secalis, which can heavily infect both rye and barley under artificial inoculation conditions. This relationship was not evaluated in our study as our sampling did not include any collections on Secale. DNA sequence data for the holotype specimen PUR 89857 grouped in Clade I, which included collections only from Elymus and Hordeum. Our results support the suggestion by Szabo (2006), that two samples from Elyt. repens (current name: Elymus repens; GRIN) were very similar to the taxon from $H$. vulgare. Fifteen host species were documented as susceptible by Jin and Steffenson (1999), including Br. tectorum, Elym. canadensis, Elym. trachycaulus, Elym. virginicus, Elym. repens subsp. repens, Thinopyrum intermedium, $H$. jubatum, $H$. vulgare, Leymus angustus, Ley. cinereus, Ley. dahuricus, Ley. racemosus, Pascopyrum smithii, Psathyrostachys juncea and Secale cereale.

7. P. coronati-japonica Liu \& Hambleton sp. nov. (Fig. 5n; Clade VIII; MycoBank \#: MB563546)

Puccinia coronata affinitis sed teleosporis hilis attenuatis et septis constrictis.

Holotypus: on Calamagrostis arundinacea, Japan, Yamanashi, Shoshenkyo, Prov. Kahi, 01 Nov. 1958, Y. Morimoto (PUR F16131).

Etymology: Refers to the known distribution of the species.

Uredinia not observed. Telia predominantly on abaxial, almost no infection on the adaxial surface, $0.3-0.5 \times$ $0.1 \mathrm{~mm}$, mostly cylindrical, not loculated; telio-paraphyses rarely observed, cylindrical; teliospores clavate, some with very long lower cells, severely constrict at septum (Fig. 5n), $36-58 \times 12-18 \mu \mathrm{m}$; the longest digitations per spore (3) $4-$ 13 (18) $\mu \mathrm{m}$; pedicel hila narrow 3.5-6.5 $\mu \mathrm{m}$.

Alternate host: Berchemia pauciflora.

Distribution: Japan.

Discussion: Telia occur mainly on the abaxial leaf surface, a striking feature shared only with one other species, $P$. coronati-calamagrostidis, which differs in having telia less frequently loculated, longer teliospores and longer 
digitations. Teliospores are severely constricted at the septum as compared to most species in the Series, except for $P$. coronati-agrostidis and $P$. coronata s.str. Considering host and geographic distribution, this species correlates well with P. coronata var rangiferina (Cummins 1971), which is also found on Cal. arundinacea and distributed in Japan. Although we obtained the type specimen of $P$. coronata var. rangiferina from PUR, we were not successful in obtaining DNA sequence data so this relationship was not evaluated with molecular characters. But our comparative morphological study revealed obvious morphological discrepancies between the two, and therefore, P. coronatijaponica is recognized as distinct, having longer teliospores and shorter, unbranched digitations. Differences in urediniospore size could not be evaluated because the two specimens analysed in this study lacked uredinia.

8. P. coronati-longispora Liu \& Hambleton sp. nov. (Figs. 5a, e; Clade II; MycoBank \#: MB563542)

Teleosporis longis et attennuatis, 34-68 $(-75) \times 10-17$ $(-19) \mu \mathrm{m}$, frequenter tricellularis, in Bromus congit.

Holotype: on Bromus erectus, Czech Republic, Hnojnice, 'České Středohoři', 31 Aug. 2002, J. Marková (PRC 196).

Etymology: Refers to the longer size of teliospores compared to P. coronati-brevispora.

Uredinia often generate light infections, adaxial, 0.3$0.4 \times 0.1 \mathrm{~mm}$, ruptured; spores $18-24(26) \times 16-23 \mu \mathrm{m}$, germ pores 9-14 scattered, cuticular caps clear in congored stain. Telia amphigenous or adaxial, $0.2-0.8 \times 0.1-$ $0.2 \mathrm{~mm}$, fusiform, the ones on abaxial side often covered (Fig. 5a), not to slight loculated; telio-paraphyses not observed; teliosopores clavate, some very long, slightly constrict to constrict at septum (Fig. 5e), 3- celled spores common, 34-68 (75) $\times 10-17$ (19) $\mu \mathrm{m}$, digitations tuberculate, cylindrical or bifurcate, the longest per spore 5-12 (14) $\mu \mathrm{m}$, pedicel hila $3.5-7 \mu \mathrm{m}$.

Host: Bromus erectus.

Alternate host: Rhamnus saxatilis.

Distribution: Slovakia, Czech Republic.

Other specimen examined: Slovakia, Bratislava, on Bromus erectus, 16, June 2002, K. Bacigalov PRC 194).

Discussion: The much longer mean length of the teliospores and the presence of 3-celled teliospores can be used to distinguish $P$. coronati-longispora from $P$. coronati-brevispora, the two species specialized on Bromus (see Tables 4, 5). However, teliospore and pedicel hilum widths are similar for both species but narrower than for the type species.

The variety "P. coronata bromi" was first recognized on Br. ciliatus in Canada by Fraser and Ledingham (1933). Later, Cummins (1971) combined it with $P$. coronata var. coronata. In Europe, multiple Bromus species including $B r$. erectus and $B r$. inermis (Mühlethaler 1911; Treboux 1912) were reported as hosts, leading Mühlethaler (1911) to designate a forma specialis for crown rust on Bromus. However, Urban and Marková (1993) disagreed and considered that the taxon on Bromus belonged to $P$. coronata var. coronata or to $P$. coronata var. avenae f. sp. graminicola.

A new morphotype (WPc-95A) on Br. inermis was discovered from Wisconsin, South Dakota and Minnesota, USA (Delgado et al. 2001; Anikster et al. 2003). Delgado et al. (2001) noted that WPc-95A was distinguishable from "P. coronata bromi" (discussed above) on the basis that the Canadian form did not infect $\mathrm{Br}$. inermis. In addition, Anikster et al. (2003) observed differences between WPc95A and three other varieties of Fraser and Ledingham (1933) and P. coronata var. hordei by Jin and Steffenson (1999). They also speculated that the new morphotype was different from $P$. coronata f. sp. bromi sensu Mühlethaler based on the evidence that WPc-95A has smaller teliospores. Results of our DNA analyses, in which samples on $B r$. inermis from North America grouped in a clade separate from those on $\mathrm{Br}$. erectus from Slovakia and the Czech Republic were supported by differences in teliospore length (Table 4, 5). We recognize the North American taxon with shorter teliospores, P. coronati-brevispora, as a new species distinct from $P$. coronati-longispora from eastern Europe. Interestingly, a specimen from Germany on $\mathrm{Br}$. erectus (BPI 718354) is more closely related to the North American variety in our ITS tree and statistical tests indicate that teliospore length is significantly shorter than that measured for the European taxon. This suggests that the species on $B r$. erectus may have diverged in Europe before the lineage migrated to North America. However, this hypothesis must be tested with comprehensive sampling (in term of genes and specimens) before drawing any conclusions.

In our study, a collection of crown rust on Br. ciliatus from Canada (DAOM 204923), presumably belonging to Puccinia coronata var. bromi Fraser and Ledingham, grouped in Clade VI, and not in either of the clades on Bromus discussed above. Clade VI comprises specimens mainly on Cal. canadensis, suggesting synonymy with $P$. coronata var. calamagrostis Fraser \& Ledingham. In Szabo's molecular analysis (2006), a single specimen on Cal. canadensis formed a unique lineage considered to be a new species (PcSP4), while both Cummins (1971) and Urban and Marková (1993) lumped collections on this host in $P$. coronata var. coronata. Our data supports Fraser and Ledingham's concept of segregating $P$. coronata var. calamagrostis, but in combination with $P$. coronata var. bromi Fraser \& Ledingham, from P. coronata var. coronata. 


\section{Unresolved lineages:}

In addition to $P$. coronata var. coronata, $P$. coronata var. avenae and $P$. coronata var. rangiferina, Cummins (1971) accepted two more varieties, $P$. coronata var. gibberosa and $P$. coronata var. himalensis.

P. coronata Corda var. gibberosa (Lagerh.) Joerst. Avh. Norske Videnskaps-Akad. Oslo I. 1948:9. 1949 (Clade XI): on Festuca altissima All., Europe, Type: Lagerheim, near Frieburg in Baden, German. Type seen!

Puccinia coronata var. gibberosa on Fe. alitissima, distributed in Europe, is reported to have teliospores with poorly defined digitations although Cummins (1971) cast doubt about the value of the "gibberose" character of the spore apex. In this study, we have shown that the shape and length of digitations combined with other characters can be useful for differentiating species. Within Series Coronata, few species are poorly digitated ( $P$. coronati-calamagrostidis), or have short digitations (P. coronati-agrostidis and P. coronati-japonica). Unfortunately, we were unable to conduct DNA analysis for the type specimen of $P$. coronata var. gibberosa (S F30373) but based on our assessment, it is morphologically similar to the type specimen of $P$. coronata var. intermedia (BRNM 111952), a variety recognized by Urban (1967). The ITS sequence of the latter specimen grouped with a PUR specimen (PUR F17485) on Fe. altissima from Slovakia, suspected of belonging to $P$. coronata var. gibberosa because of the same host and origin. Based on this evidence, we hypothesize that the two taxa are synonymous.

P. coronata Corda var. himalensis Barclay Trans. Linn. Soc. Lodon 3:227. 1891 (Clade X): on Calamagrostis canadensis, Japan, Type: on Brachypodium sylvaticum, India, Simla (K), by Barclay. Type not seen.

Puccinia coronata var. himalensis was characterized by having small thin-walled urediniospores and exposed aparaphysate telia. Multiple previously accepted species were included as synonyms including $P$. brevicornis, $P$. melicae, and $P$. subdigitata (see Results and discussion section and Table 1 for citation and voucher information) by Cummins (1971). Based on analyses of ITS sequences, type specimens sampled for these synonyms did not form a monophyletic group indicating that they are not conspecific.

Acknowledgments This study was part of a broader project (CRTI040045RD) funded by the Canadian Chemical, Biological, RadiologicalNuclear, and Explosives (CBRNE) Research and Technology Initiative (CRTI). We thank all the Herbaria for kindly providing specimens for our research: B, BP, BPI, BR, BRNM, DAOM, HMAS, K, PRC, PRM, PUR, and $\mathrm{S}$. We also appreciate the assistance of Cathie Aime, Tom Fetch, Shelin Jin and Chunjie Li for help with obtaining new collections from the field, and Elisa McCabe, Rafik Assabgui and Julie Chapados for DNA sequencing technical support. In particular, we are grateful to Dr. J. A. Parmelee and Dr. S. Redhead for mentorship of ML and SH in rust taxonomy and advice on nomenclatural issues.
Open Access This article is distributed under the terms of the Creative Commons Attribution License which permits any use, distribution, and reproduction in any medium, provided the original author(s) and the source are credited.

\section{References}

Anikster Y, Eilam T, Manisterski J, Leonard KJ (2003) Self-Fertility and Other Distinguishing Characteristics of a New Morphotype of Puccinia coronata Pathogenic on Smooth Brome Grass. Mycologia 95:87-97

Ayliffe MA, Dodds PN, Lawrence GJ (2001) Characterisation of a beta-tubulin gene from Melampsora lini and comparision of fungal beta-tubulin genes. Mycol Res 105:818-826

Baker RH, Yu X, DeSalle R (1998) Assessing the Relative Contribution of Molecular and Morphological Characters in Simultaneous Analysis Trees. Mol Phylog Evol 9:427-436

Brown MR (1937) A study of crwn rust, Puccinia coronata Corda, in Great Britain. I. Physiologic specialization in the uredospore stage. Ann Appl Biol 24:504-527

Carsten LD, Johnston MR, Douglas LI, Sands DC (2000) A Field Trial of Crown Rust (Puccinia coronata f. sp. avenae) as a Biocontrol Agent of Wild Oats on San Clemente Island. Biol Control 19:175181

Corda ACJ (1837) Icones fungorum hucusque cognitorum 1:6. Abbildungen der Pilze und Schwaemme. Apud. JG Calve, Prague

Cornu M (1880) Note sur les generations des Uredinees. Bull Soc Bot Fr 27:179

Cummins GB (1971) The rust fungi of cereals, grasses and bamboos. Springer-Verlag, New York

Delgado NJ, Grau CR, Casler MD (2001) Host Range and Alternate Host of a Puccinia coronata Population from Smooth Brome Grass. Plant Dis 85:513-516

Dietz S (1926) The alternate hosts of crown rust, Puccinia coronata Corda. J Agri Res 33:953-970

Eriksson J (1894) Uber die specializierung des parasitismus bei den Getreiderostpilzen. Berichte des deutschen botanischen Gesellschatt 12:292-331

Eriksson J (1908) Neue Studien über die spezielisierung der grasbewohnenden Kronenrostarten. Arkiv Bot 8:1-26

Eshed N, Dinoor A (1980) Genetics of pathogenicity in Puccinia coronata: pathogenic specilzation at the host genus level. Phytopathology 70:1042-1046

Fraser WP, Ledingham GA (1933) Studies of the crown rust, Puccinia coronata Corda. Sci Agri 13:313-323

Hall TA (1999) BioEdit: a user-friendly biological sequence alignment editor and analysis program for Windows 95/98/NT. Nucl Acids Symp Ser 41:95-98

Harder DE, Haber S (1992) Oat disease and pathologic techniques. In: Matshall HG, Sorrells ME (eds) Oat science and technology. American Society of Agronomists, Inc. and Crop Science Soceity of America, Inc, Madison

Huelsenbeck JP, Ronquist F (2001) MRBAYES: Bayesian inference of phylogeny. Bioinformatics 17:754-755

Jin Y, Steffenson BJ (1999) Puccinia coronata var. hordei var. nov.: Morphology and pathogenicity. Mycologia 91:877-884

Katoh K, K-i K, Toh H, Miyata T (2005) MAFFT version 5: improvement in accuracy of multiple sequence alignment. Nucl Acids Res 33:511-518

Kaufman MJ (1967) The species of Uredinales on Bromus. Mycopathologia 32:249-261

Klebahn H (1892) Kulturversuche mit heterocischen Uredineen. Z Pflanzenkr Galenkd 2:332-343 
Klebahn H (1894) Kulturversuche mit heteröcischen uredineen. Zeitschrift für Pflanzenkrankheiten 4:129-139

Kornerup A, Wanscher JH (1967) Methuen handbook of colour, 2nd edn. Methuen, London

Liu M, Hambleton S (2010) Taxonomic study of stripe rust, Puccinia striiformis sensu lato, based on molecular and morphological evidence. Fungal Biol 114:881-899

Liu Y, Whelen S, Hall BD (1999) Phylogenetic relationhips among Ascomycetes: evidence from an RNA polymerase II subunit. Mol Biol Evol 16(12):1799-1808

McCallum BD, Fetch T, Chong J (2007) Cereal rust control in Canada. Aust J Agri Res 58:639-647

Melhus I, Dietz S, Willey F (1922) Alternate hosts and biologic specialization of crown rust in America. Iowa Agri Exp Sta Res Bull 49:115-144

Muhlethaler F (1911) Infektionsversuche mit Rhmanus befallended Kronenrosten. Zentralblatt für Bakteriologie $2 \mathrm{Abt}$ 30:386-419

Nielsen P (1877) Bemaerkningen om nogle Rustarter. Botanisk Tidsskrift udgivet af den Botaniske Forening i Kjobenhavn 3:39

Peturson B (1954) The relative prevalence of specialized forms of Puccinia coronata that occur on Rhamnus cathartica in Canada. Can J Bot 32:40-47

Posada D, Crandall KA (1998) Modeltest: testing the model of DNA substitution. Bioinformatics 14:817-818

Potter LR, Caga B, Paul BVH, Birckenstaedt E (1990) Pathogenicity of Some European Collections of Crown Rust (Puccinia coronata Corda) on Cultivars of Perennial Ryegrass. J Phytopathol 130:119-126
Roelfs AP (1985) Epidemiology in North America. In: Roelfs AP, Bushnell WR (eds) Cereal Rusts. Acamedic Press, New York, pp 403-434

Simons M (1970) Crown rust of oats and grasses. Monograph No. 5. The American Phytopathological Society, APS Press, St Paul

Smith JF (2000) Phylogenetic signal common to three data sets: Combining data which initially appear heterogeneous. Plant Syst Evol 221:179-198

Sorenson MD, Franzosa EA (2007) TreeRot, Version 3. Boston University, Boston

Swofford DL (1998) PAUP*. phylogenetic analysis using parsimony (*and other methods). version 4 (computer program). Sinauer Associates, Sunderland

Szabo LJ (2006) Deciphering species complexes: Puccinia andropogonis and Puccinia coronata, examples of differing modes of speciation. Mycoscience 47:130-136

Treboux O (1912) Infektionsversuche mit parasitischen Pilzen III. Ann Mycol 10:557-563

Treboux O (1914) Infektionsversuche mit parasitischen Pilzen IV. Ann Mycol 12:480-483

Urban Z (1963) Nová metoda umožňující pozorování kličních porů urediospor a její použití v taxonomii travních rzí. Česká Mykologie 17:193-194

Urban Z (1967) The taxonomy of some European graminicolous rusts. Česká Mykologie 21:12-16

Urban Z, Marková J (1993) The rust fungi of grasses in Europe. I. Puccinia coronata Corda. Acta Univ Carol 37:93-147 